\title{
Eight different types of dopaminergic neurons innervate the Drosophila mushroom body neuropil: anatomical and physiological heterogeneity
}

\author{
Zhengmei Mao ${ }^{1 \dagger}$ and Ronald L. Davis ${ }^{1,2 *}$ \\ 1 Department of Molecular and Cellular Biology, Baylor College of Medicine, Houston, TX, USA \\ 2 Menninger Department of Psychiatry and Behavioral Sciences, Baylor College of Medicine, Houston, TX, USA
}

\section{Edited by:}

Liqun Luo, Stanford University, USA

Reviewed by:

Volker Hartenstein, University of

California, Los Angeles, USA

Yi Rao, Peking University, China

Tzumin Lee, University of

Massachusetts, USA

*Correspondence:

Ronald L. Davis, Department of Molecular and Cellular Biology,

Menninger Department of Psychiatry and Behavioral Sciences, Baylor

College of Medicine, One Baylor Plaza,

Houston, TX 77030, USA.

e-mail: rdavis@bcm.tmc.edu

${ }^{+}$Current address: Zhengmei Mao,

Institute of Molecular Medicine,

University of Texas Health Science

Center at Houston, Houston, TX, USA.
We examined tyrosine hydroxylase (TH-GAL4) expression and anti-TH immunoreactivity in the Drosophila protocerebrum and characterized single cell clones of the TH-GAL4 neurons. Eight clusters of putative dopaminergic neurons were characterized. Neurons in three of the clusters project to the mushroom body neuropil: PAM neurons project to the medial portion of the horizontal lobes; PPL1 neurons project to the vertical lobes, the junction area, the heel and distal peduncle; and PPL2ab neurons project to the calyx. Five types of PPL1 neurons were discovered that innervate different zones of the mushroom body lobes. Functional imaging experiments showed that the dopaminergic processes in four of the zones differ in response properties to odor, electric shock, or following the pairing of odor and electric shock. These results indicate that distinct dopaminergic neurons define separate zones of the mushroom body lobes and are probably involved in different functions. Differences in functional response properties of these neurons suggest that they are involved in different behavioral processes.

Keywords: dopamine, olfactory learning, Drosophila, single cell clones, functional optical imaging, tyrosine hydroxylase

\section{INTRODUCTION}

In mammals, the neurotransmitter dopamine (DA) modulates movement, motivation, and cognition. Abnormal functional states of the DA system in humans is thought to underlie the behavioral anomalies of Parkinson's disease, Attention Deficit Hyperactivity disorder (ADHD), and schizophrenia. In insects like Drosophila, DA is involved in locomotion (Pendleton et al., 2002), sleep and arousal (Andretic et al., 2005; Foltenyi et al., 2007; Kume et al., 2005), courtship behavior (Liu et al., 2008; Neckameyer, 1998), inhibition of startle-induced hyperexcitability (Friggi-Grelin et al., 2003), saliency-based decision making (Zhang et al., 2007), and associative learning, the latter often measured by olfactory classical conditioning using an odor as conditioned stimulus (CS). Depletion of DA or inhibition of neurotransmitter release from dopaminergic (DA) neurons impairs the formation of aversive, but not appetitive, olfactory memory (Schwaerzel et al., 2003; Tempel et al., 1984). One prominent model is that DA signals comprise the neural representation of the US or are part of a composite US representation for aversive olfactory conditioning (Davis, 2005).

In mammals, DA neurons form nine clusters localized to the mesencephalon, the diencephalon and the olfactory bulb (Björklund and Dunnett, 2007). The major output pathways include the nigrostriatal pathway from the substantia nigra to the caudate putamen, the mesolimbic pathway from the ventral tegmental area (VTA) to the limbic areas of the brain, the mesocortical pathway from the VTA to the cortex, and the tuberoinfundibular pathway from the periventricular and arcuate nuclei of the hypothalamus to the median eminence of the hypothalamus (Vallone et al., 2000). Fifteen clusters of DA neurons have been identified distributed throughout the Drosophila brain using histofluorescence and immunohistochemistry (Budnik and White, 1988; Nässel and Elekes, 1992), but the projection patterns of neurons in each cluster have not been delineated, and the specific neurons that project to the mushroom bodies, brain regions critical for learning (Davis, 2005), remain unknown.

The midbrain DA neurons in mammals respond initially during associative learning by bursts of firing to a rewarding stimulus. After learning, this activity is transferred to the CS that predicts the reward US. Aversive events produce depressions of DA neuron activity (Schultz, 2007). In flies, DA neurons would be expected to respond to the US of electric shock and not to the odor CS if DA neurons represent only the US. Consistent with this hypothesis, DA processes in the mushroom body lobes were reported to respond strongly to electric shock and weakly to odor presented to the fly. The calcium response of these processes to an odor CS became prolonged after the odor was paired with electric shock (Riemensperger et al., 2005).

We used a tyrosine hydroxylase GAL4-transgene (TH-GAL4; Friggi-Grelin et al., 2003) and single cell, genetic visualization techniques to examine the projections of individual TH-GAL4 expressing neurons in the Drosophila protocerebrum and discovered that eight different types of these neurons extend projections to the mushroom bodies. The expression pattern of the TH-GAL4 transgene 
is broadly similar but not completely identical to the expression of endogenous TH (a marker for DA neurons) as described below. Nevertheless, we refer to the TH-GAL4 positive cells as DA or putative DA neurons because this is likely the case and for simplicity, although it should be understood that being TH-GAL4 positive is not necessarily equivalent to being dopaminergic on a cell-by-cell basis. The innervation was complex, with different zones of the mushroom body neuropil being innervated by different DA neurons. We also visualized the response properties of the DA terminals in four such zones as living animals were presented with odor, electric shock, or combined odor/electric shock. The processes in different zones exhibited different response profiles, suggesting that the mushroom body lobes are subdivided into distinct zones defined by synaptic contacts from distinct DA neurons.

\section{MATERIALS AND METHODS EXPERIMENTAL ANIMALS}

The tyrosine hydroxylase GAL4 line TH-GAL4 (Friggi-Grelin et al., 2003; kindly provided by S. Birman) was used to drive UAS-mCD8:: GFP, UAS-syt.eGFP or UAS-GFP::LacZ.nls for immunohistochemistry experiments. FLP-out studies were performed using animals that carried the TH-GAL4 driver along with a heat-shock promoter driven flippase $(h s F L P)$ and the reporter $U A S>C D 2, y^{+}>C D 8:: G F P$ (kindly provided by J. Wang, Wong et al., 2002). These animals were raised at $18^{\circ} \mathrm{C}$ except for a heat-shock of generally 10-30 min at $32^{\circ} \mathrm{C}$ in a water bath or air incubator at several different larval or pupal stages. For in vivo imaging, TH-GAL4 was used to drive UAS-G-CaMP (Yu et al., 2004). These animals were maintained at room temperature. Flies were kept on standard food medium and harvested at 4 days of age for the experiments.

\section{IMMUNOHISTOCHEMISTRY}

Fly heads were fixed in phosphate buffered saline (PBS), $\mathrm{pH} 7.4$, containing 4\% paraformaldehyde and $0.3 \%$ Triton X-100 for $1 \mathrm{~h}$ at $4^{\circ} \mathrm{C}$. They were then washed with $1 \times \mathrm{PBS}, \mathrm{pH} 7.4$, containing $0.3 \%$ Triton $\mathrm{X}-100$ for $3 \times 10 \mathrm{~min}$ at $4^{\circ} \mathrm{C}$. Cuticle and trachea were removed and brains were washed again for $3 \times 10 \mathrm{~min}$. Samples were incubated in blocking buffer $(5 \%$ normal goat serum in $1 \times$ PBS, pH 7.4, containing $0.3 \%$ Triton $\mathrm{X}-100$ ) for $2 \mathrm{~h}$ at $4^{\circ} \mathrm{C}$. They were then incubated with primary antibody diluted in blocking buffer overnight at $4^{\circ} \mathrm{C}$. After washing for $6 \times 10 \mathrm{~min}$ at $4^{\circ} \mathrm{C}$, the samples were incubated with secondary antibody diluted in blocking buffer overnight at $4^{\circ} \mathrm{C}$. They were washed $6 \times 10 \mathrm{~min}$ at $4^{\circ} \mathrm{C}$ and mounted in Vectashield (Vector Laboratories). The primary antibodies used include rabbit anti-GFP (1:400, Molecular Probes), mouse anti-tyrosine hydroxylase (1:200, Chemicon), mouse antiFasII (1:2, DSHB), mouse anti-nc82 (1:200, kindly provided by M. Heisenberg), mouse anti-beta galactosidase (1:100, DSHB), and rat anti-mouse CD8 (1:100, Caltag). Secondary antibodies were goat anti-rabbit IgG and goat anti-mouse or -rat IgG conjugated with Alexa Fluor 488, 568 or 633 (Molecular Probes, all at 1:500). TOTO-3 (Molecular Probes) was added to the secondary antisera at a 1:500 dilution to stain nuclei. Images were collected using a $20 \times$ dry or a $63 \times$ water immersion objective of a Leica TCS SP1 or SP 5 confocal microscope. The step size for $\mathrm{z}$-stacks during imaging was typically $1 \mu \mathrm{m}$ or less, with images collected typically at $1024 \times 1024$ pixel resolution. Over 2000 brains were analyzed for TH-GAL4 clones.

\section{FUNCTIONAL IMAGING}

The procedures used here were similar to those described in Yu et al. (2004). Flies carrying TH-GAL4>UAS-G-CaMP were mounted in pipette tips sculpted to cradle the fly's body but expose the dorsal aspect of the head and the proboscis. The head and scutum were immobilized to the pipette tip with myristic acid. A small area of cuticle from the dorsal head along with the underlying trachea was removed and the opening was covered with a small piece of plastic wrap. A low toxicity silicone adhesive (KWIK-SIL, from WPI) was used to seal the edges of the cover to the head. Flies were imaged using a $20 \times$ objective of a Leica TCS SP1 confocal microscope. G-CaMP fluorescence was produced using the $488 \mathrm{~nm}$ line of an argon laser. Time stacks were recorded at 4.4 frames/s.

Odorants were diluted in $4 \mathrm{ml}$ of mineral oil contained in a $20 \mathrm{ml}$ scintillation vial. Each odor was presented to the fly with a puffer pipette carrying the odor in an air stream by wafting pressurized air over the odorant. During an experiment, each animal was presented with a constant flow of fresh air and then odor was introduced using a three-way solenoid valve connected to a programmable controller such that there was no significant fluctuation in total air flow $(100 \mathrm{ml} / \mathrm{min})$ when switching between fresh air and air laced with odor. All animals were pre-tested in a mock odor stimulation trial using a fresh, empty vial substituted for the vial containing odor in order to exclude those animals that had become sensitized to mechanical stimulation. Odor was presented to the fly for $3 \mathrm{~s}$ when testing odor responses or for $1 \mathrm{~min}$ when paired with electric shock for conditioning. Electric shock was applied via electrodes made of $50 \mu \mathrm{m}$ platinum/iridium wire that contacted the legs of the fly and also touched the abdomen if the legs were positioned near the abdomen. Constant current pulses were generated by a S48 stimulator connected to a PSIU6 stimulus isolation unit (Grass Technologies). For conditioning experiments, animals were imaged during a $3 \mathrm{~s}$ odor stimulation prior to conditioning and during another $3 \mathrm{~s}$ odor stimulation after conditioning. To be certain that electric shock was delivered to the fly, the current in the circuit was recorded with an amphere meter during each pulse of electric shock. To be certain that each fly retained responsiveness throughout the experiment, an additional puff of odor was presented to each fly at the end of experimentation. An additional train of 12 electric shock pulses was also presented to flies that received shock during conditioning, namely the forward, backward and US only groups. Only flies exhibiting a response to these post-experimental control procedures were used in the final data to exclude those flies that had become non-responsive to sensory stimuli due to inviability or other factors.

\section{DATA ANALYSIS}

The analysis of functional imaging data was performed as described by Yu et al. (2004). A region of interest (ROI) was identified and the average pixel intensity in the ROI across the time stack was obtained. Baseline fluorescence $\left(F_{\mathrm{o}}\right)$ was calculated as the average pixel intensity in the ROI over five successive frames immediately prior to the delivery of odor or electric shock. The peak fluorescence $(F)$ was calculated as the maximum average intensity over five successive frames during the presentation of odor or electric shock. The percent change in fluorescence during stimulation (Percent $\Delta F$ ) was calculated as $\Delta F=\left(F-F_{\mathrm{o}}\right) / F_{\mathrm{o}}$. Statistical comparisons were performed 
using ANOVA followed by Bonferroni/Dunn post hoc tests. For the presentation of pseudocolor images, each raw image was passed through a median filter with a radius of 3 pixels. Five successive frames each for $F$ and $F_{0}$ were averaged, and the percent $\Delta F$ image was calculated for each pixel using ImageJ. Immunohistochemistry data were visualized with Leica LAS AF software and ImageJ.

\section{RESULTS}

\section{TH-GAL4 POSITIVE NEURONS IN THE ADULT PROTOCEREBRUM}

We used the TH-GAL4 transgene to drive the expression of UAS$m C D 8:: G F P$ to identify and further characterize putative DA neurons in the adult protocerebrum from whole mount specimens. Although there exist numerous DA neurons scattered throughout the adult fly brain, we restricted our study to these neurons with cell bodies residing in the protocerebrum.
Figures 1A,D illustrate anterior and posterior projection views respectively of the $T H$-GAL4-positive cells and their broadly distributed processes. Careful visual inspection of multiple sets of high resolution stacks allowed us to identify cell bodies and to resolve their primary processes in most cases. Cells of a similar size, shape, location, and projection pattern of the primary process were recognized as class. Some classes consisted of one single neuron; others consisted of a cluster of neurons. The primary processes of neurons in the same cluster frequently fasciculated together near their cell bodies (Figure 1D). Figures 1B,E illustrate the cell bodies in the protocerebrum and their class affiliation. Figures 1 C,F present a schematic diagram of the location of the various classes.

We observed approximately $127 \mathrm{TH}-\mathrm{GAL} 4$ expressing cells that can be categorized into 13 different classes scattered on the anterior and posterior faces of the protocerebrum (Figure 1; Table 1). The classes

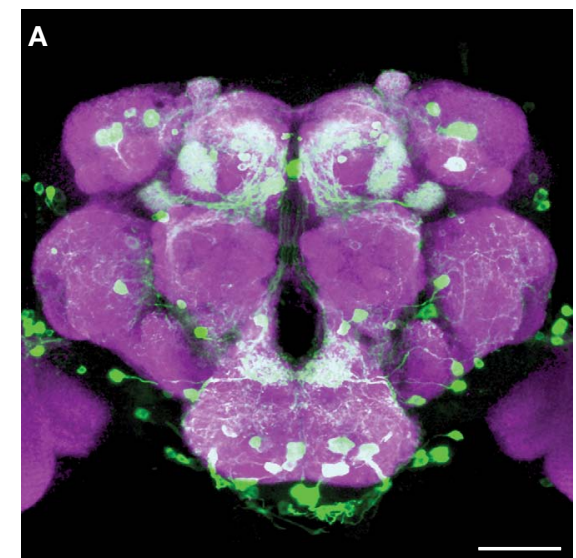

B

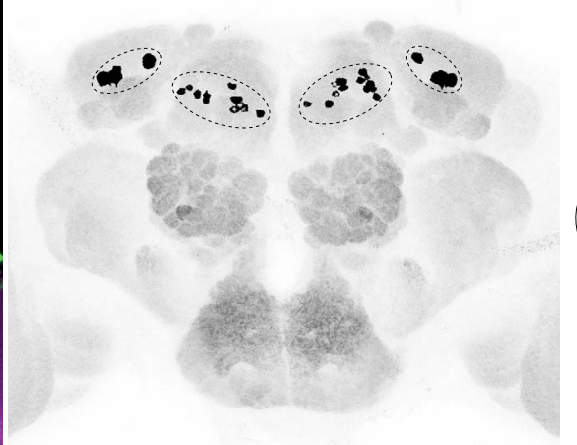

C

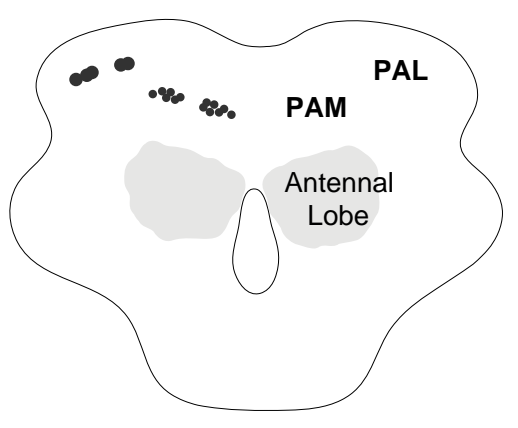

$\mathbf{F}$

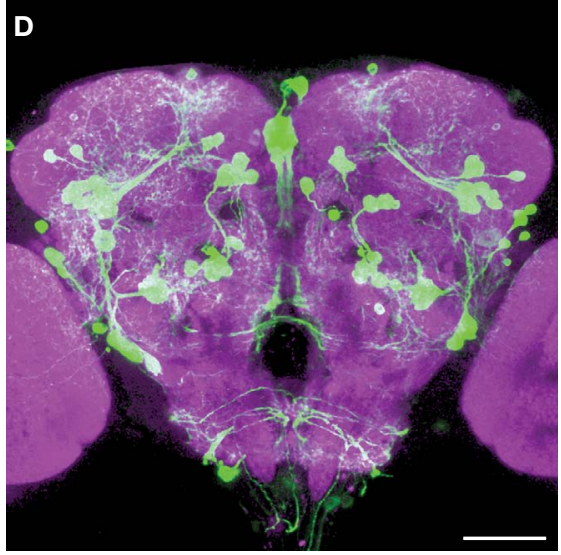

E

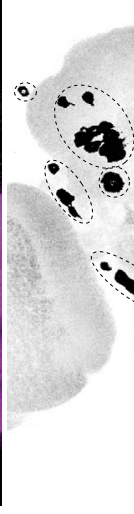

FIGURE 1 | TH-GAL4 expressing neurons in the Drosophila brain as visualized with TH-GAL4>UAS-mCD8::GFP. (A-C) Anterior view. (D-F) Posterior view. (A,D) Anti-GFP staining (green) showing cell bodies and processes of TH-GAL4 expressing cells. The brain was counterstained (magenta) with an anti-nc82 antibody. Scale bar, $50 \mu \mathrm{m}$. (B,E) The cell bodies were identified in the protocerebrum by thresholding images taken from the brains shown in panels (A) and (D). Dotted lines encircle groups of cells. $(\mathbf{C}, \mathbf{F})$ Schematic diagram of $(\mathbf{B}, \mathbf{E})$ indicating the name, location and typical number of TH-GAL4 expressing cell clusters in the protocerebrum (Tanaka et al., 2008). The protocerebral anterior medial (PAM) cluster resides near the anterior face of the brain in the anterior inferiormedial protocerebrum. The protocerebral anterior lateral (PAL) cluster is lateral to the dorsal portion of the vertical lobes in the middle superiorlateral protocerebrum (Figure 3A) The protocerebral posterior medial (PPM) clusters all reside on the posterior face of the brain in the posterior superiormedial protocerebrum (PPM1), the posterior inferiormedial protocerebrum (PPM2) and the superior posterior slope (PPM3, PPM4). Similarly, the protocerebral posterior lateral (PPL) clusters are also near the posterior face of the brain in the posterior inferiorlateral protocerebrum (PPL1), the posterior lateral protocerebum (PPL2c, PPL4, and PPL2ab), or near the boundary of the superior posterior slope and the posterior lateral protocerebrum (PPL3). The PPL5 neuron is located near the dorsal edge of the lateral horn. The PPD neuron is located at the dorsal surface of the brain, posterior to the tips of the vertical lobes. 
were bilaterally symmetrical in each hemisphere except for PPM1, which was positioned along the dorsal/ventral midline. Although they differed from one another in the average number of cells that they contain (from 1 to 13; Table 1), most individual classes contained a highly reproducible number of neurons between brains (Table 1).

Based on cell body location and subsequent confirmation of anti-tyrosine hydroxylase immunoreactivity (Figures 2 and 3C), eight of the clusters we identified appear to correspond to the seven groups of DA neurons in the blowfly and Drosophila identified by Nässel and Elekes (1992). The PPL2c neurons, present in Drosophila but not blowfly, were previously designated as a subgroup of the PPL2 group. We placed them in a separate cluster based on the considerable distance between the cell bodies of these neurons and the PPL2ab neurons and more importantly, on the dissimilar projection pattern of these neurons (Figure 2 in Supplementary Material) compared to the PPL2ab neurons (Figure 6) as revealed by clonal analysis (see below).

TH-GAL4 was also expressed in five additional classes that were not previous identified. These classes, mostly contained one single neuron, failed to stain for tyrosine hydroxylase (Figure 2) and are not likely to be DA neurons.

\section{ANTI-TH POSITIVE NEURONS IN THE ADULT PROTOCEREBRUM}

Of the eight TH-GAL4 positive clusters that also showed anti-TH immunreactivity (Table 1), five appeared to exhibit coincidental expression of TH-GAL4 and TH albeit with variable intensities on a cell-by-cell basis. Three clusters, PAM, PPM1 and PPL2c, exhibited a noticeable difference in the expression of the two markers. The

Table 1 | Classes of TH-GAL4 and anti-TH positive neurons in the protocerebrum.

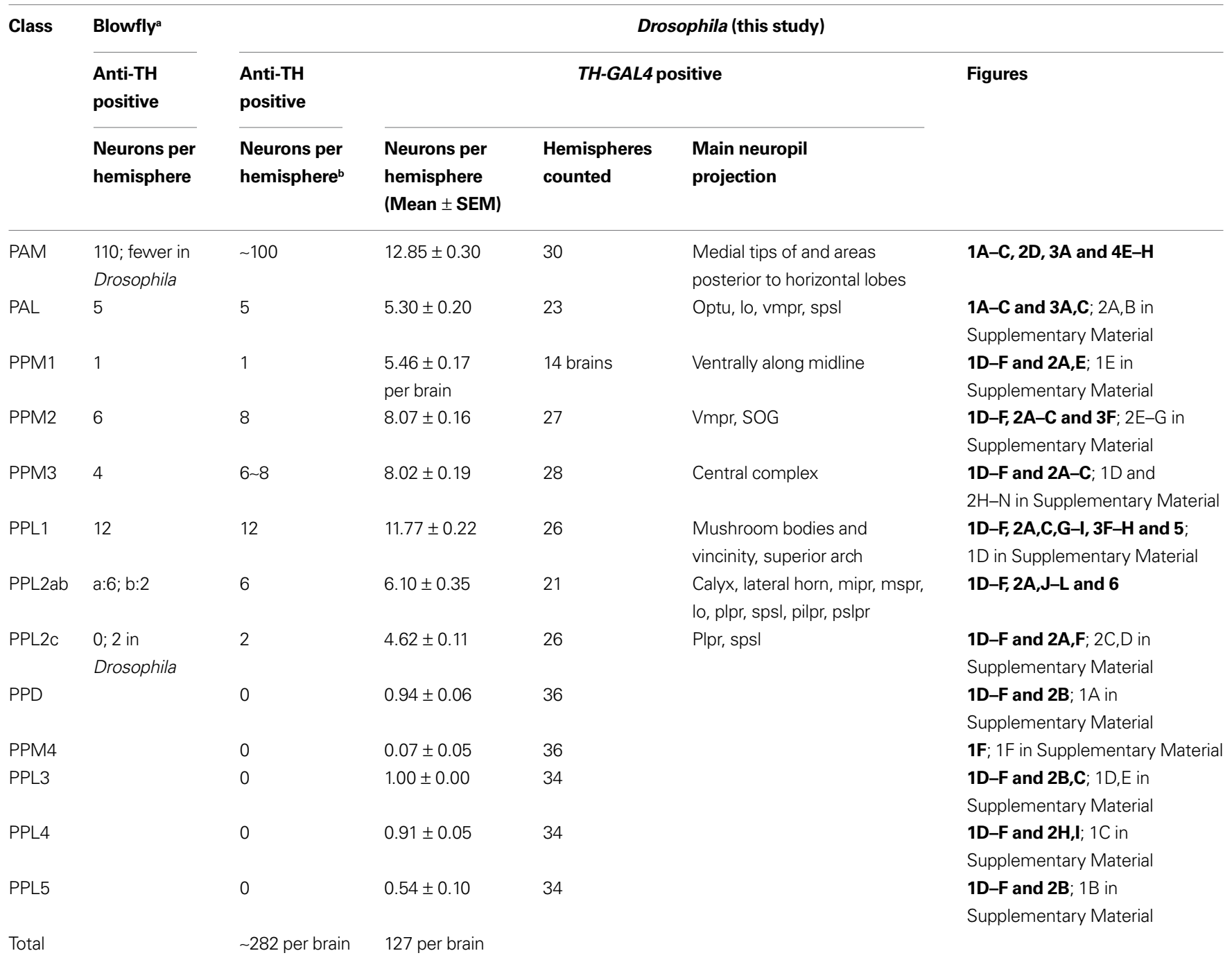

10, Lobula; mipr, medial inferior protocerebrum; mspr, medial superior protocerebrum; optu, optic tubercle; pilpr, posterior inferior lateral protocerebrum; plpr, posterior lateral protocerebrum; pslpr, posterior superior lateral protocerebrum; SOG, subesophageal ganglion; spsl, superior posterior slope; vmpr, ventral medial protocerebrum (Tanaka et al., 2008).

aNässel and Elekes (1992) documented the number of neurons in each class in blowfly and indicated that the number are homologous in Drosophila except for the two differences noted.

${ }^{b}$ Number of hemispheres counted: $\geq 6$ for each class. 

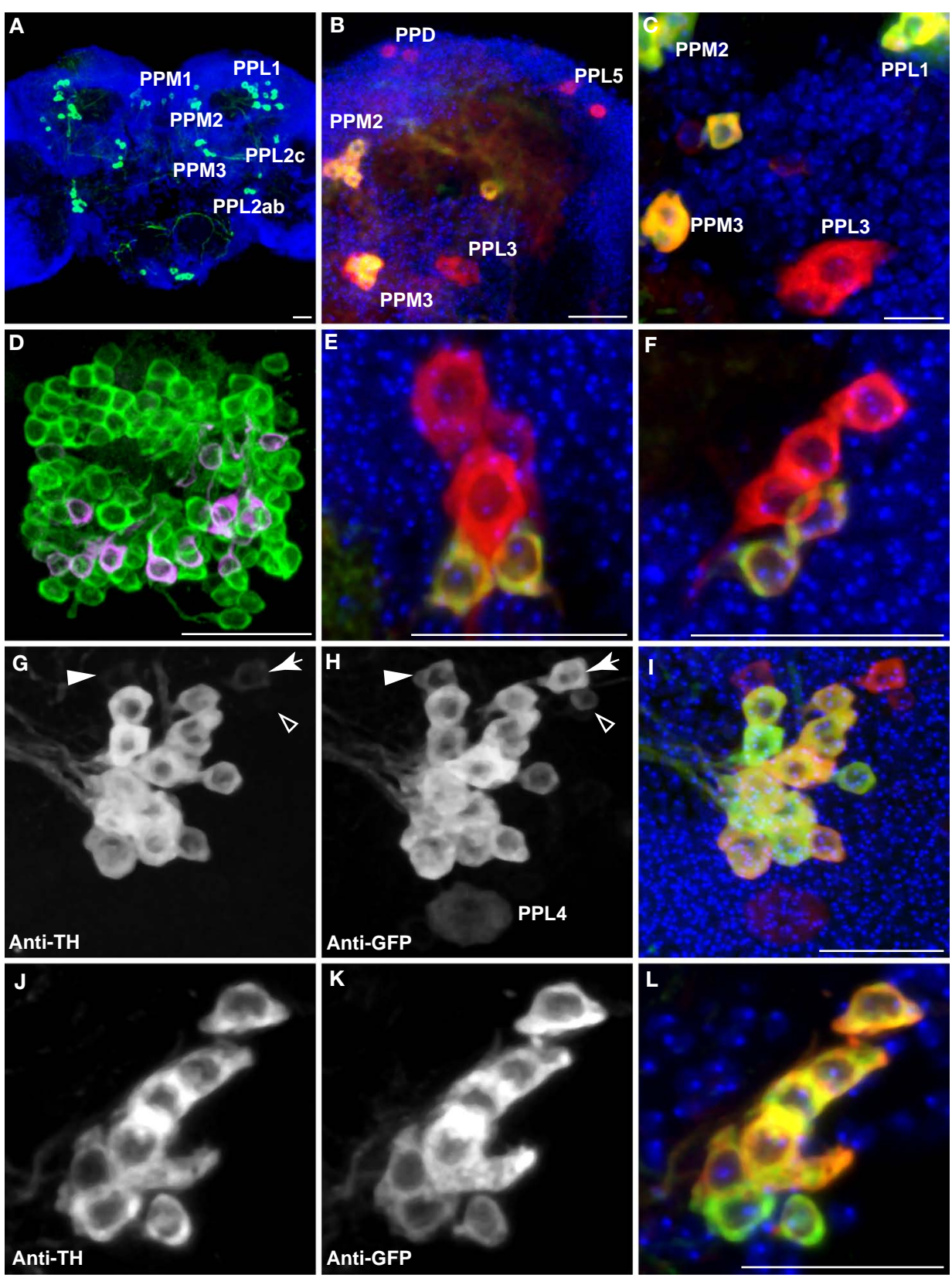

FIGURE 2 | Dopaminergic neurons as identified by anti-TH staining compared with those identified by TH-GAL4>UAS-mCD8::GFP expression. Medial is left in all panels except (A) and (E) which shows both hemispheres: dorsal is up in all panels. Panel (D) is an anterior view. All others are viewed from a posterior perspective. Scale bar in all panels: $20 \mu \mathrm{m}$. (A) Six clusters of neurons (green) were positive for anti-TH in the posterior protocerebrum. These correspond to the clusters PPM1, PPM2, PPM3, PPL1, PPL2c, and PPL2ab

(Figure 1). Blue, TOTO-3 staining cell nuclei in the brain. (B) In addition to the six clusters of cells identified in (A), TH-GAL4>UAS-mCD8::GFP expression was also observed in four groups of cells that are TH-negative, labeled as PPD, PPL5, and PPL3 in this panel and PPL4 in panel (H). Green, anti-TH immunostaining. Red, TH-GAL4>UAS-mCD8::GFP, identified with an anti-GFP antibody unless otherwise noted. Blue, TOTO-3. (C) The PPL3 cell is one large neuron lateral to the PPM3 cluster (partially shown) and ventromedial to the PPL1 group (partially shown). (D) High magnification view of the PAM cluster. PAM (Figure 1) neurons positive for anti-TH (green) far outnumber those that express
TH-GAL4>UAS-mCD8::GFP (magenta). All cells identified with TH-GAL4>UAS$m C D 8:: G F P$ were also anti-TH positive. (E) For PPM1, anti-TH stains two small cells, while TH-GAL4 expresses in additional large cells. (F) For PPL2c, anti-TH stains only two cells, while TH-GAL4 typically expresses in three additional cells. (G-I) Anti-TH immunostaining (G) and anti-GFP immunostaining (H) of the PPL1 group. (I) is an overlay of (G) and (H) with TOTO-3 staining. All anti-TH positive cells are also labeled by TH-GAL4. TH-GAL4 may express in one or two additional cells. Arrow: a cell that is strongly labeled by TH-GAL4 but only faintly by anti-TH. Arrowhead: a cell that is modestly labeled by $T H-G A L 4$ but faintly by anti-TH. Open arrowhead: one of the small cells labeled by TH-GAL4 that are scattered in several areas on the surface of the brain. This cell is not counted as a PPL1 neuron. The PPL4 neuron is one large cell ventral to the PPL1 cluster. It is only labeled by TH-GAL4 ((H), also Figure 1) but not anti-TH. (J-L) Anti-TH immunostaining $(\mathbf{J})$ and anti-GFP immunostaining (K) of the PPL2ab group. (L) is overlay of (J) and (K) with TOTO-3 staining. The same set of cells is labeled by both anti-TH and TH-GAL4. 
number of cells in the PAM cluster labeled by the anti-TH antibody far exceeded that identified by the TH-GAL4 driver (Figure 2D; Table 1) as noted by Friggi-Grelin et al. (2003). Although the TH-GAL4 driver identified only 13 PAM cells per hemisphere, we counted about 100 cells per hemisphere in this cluster that were anti-TH immunoreactive (Figure 2D; Table 1). In the PPM1 cluster, two cells showed anti-TH immunoreactivity but five cells were labeled by TH-GAL4 (Figure 2E; Table 1). The three additional cells labeled by TH-GAL4 only were larger than the two that were also positive for anti-TH immunoreactivity. There were five positive $T H-G A L 4$ neurons in the PPL2c cluster, but only two of these exhibited detectable expression of TH (Figure 2F; Table 1). In addition, the variable expression of both markers between labeled cells within some of the larger clusters made difficult an assessment of absolute co-expression of TH-GAL4 and anti-TH immunoreactivity. For instance, 1-2 of the cells within the PPL1 cluster sometimes exhibited very weak anti-TH immunoreactivity compared to the TH-GAL4 signal (Figures 2G-I). Smaller or more isolated clusters were easier to evaluate for coincidental expression (Figures 2J-L). Overall, the TH-GAL4 driver offers a good surrogate label and driver for DA neurons with the exceptions noted above.

\section{DA INNERVATION OF THE MUSHROOM BODIES}

Previous studies have shown that the mushroom body neuropil is innervated by DA terminals, consistent with the finding that DA receptors are enriched in the mushroom bodies (Han et al., 1996; Kim et al., 2003). Nässel and Elekes (1992) observed anti-DA and anti-TH reactivity in the lobes and parts of the peduncles, but not the calyx, of blowflies. High expression of TH-GAL4 or TH has also been observed in the Drosophila mushroom body lobes and heel, but not in calyx (Schwaerzel et al., 2003; Zhang et al., 2007). We re-examined the expression of both TH and TH-GAL4 in the mushroom bodies. Our findings are consistent with prior literature except that we also discovered expression of both markers in the calyx.

$T H$-GAL4 was highly expressed in the mushroom body vertical lobes, and the lateral portion of the horizontal lobes (Figures $3 \mathbf{A}, \mathbf{B}$ ). There was no significant TH-GAL4 expression in other areas of the horizontal lobes except for sparse fibers that wrap around the distal tip of the horizontal lobes. We observed dense arborizations and synaptic puncta in the area between the vertical and the horizontal lobes, an area sometimes referred to as the mushroom body satellite area (Figures 3A,B). TH-GAL4 was also strongly expressed
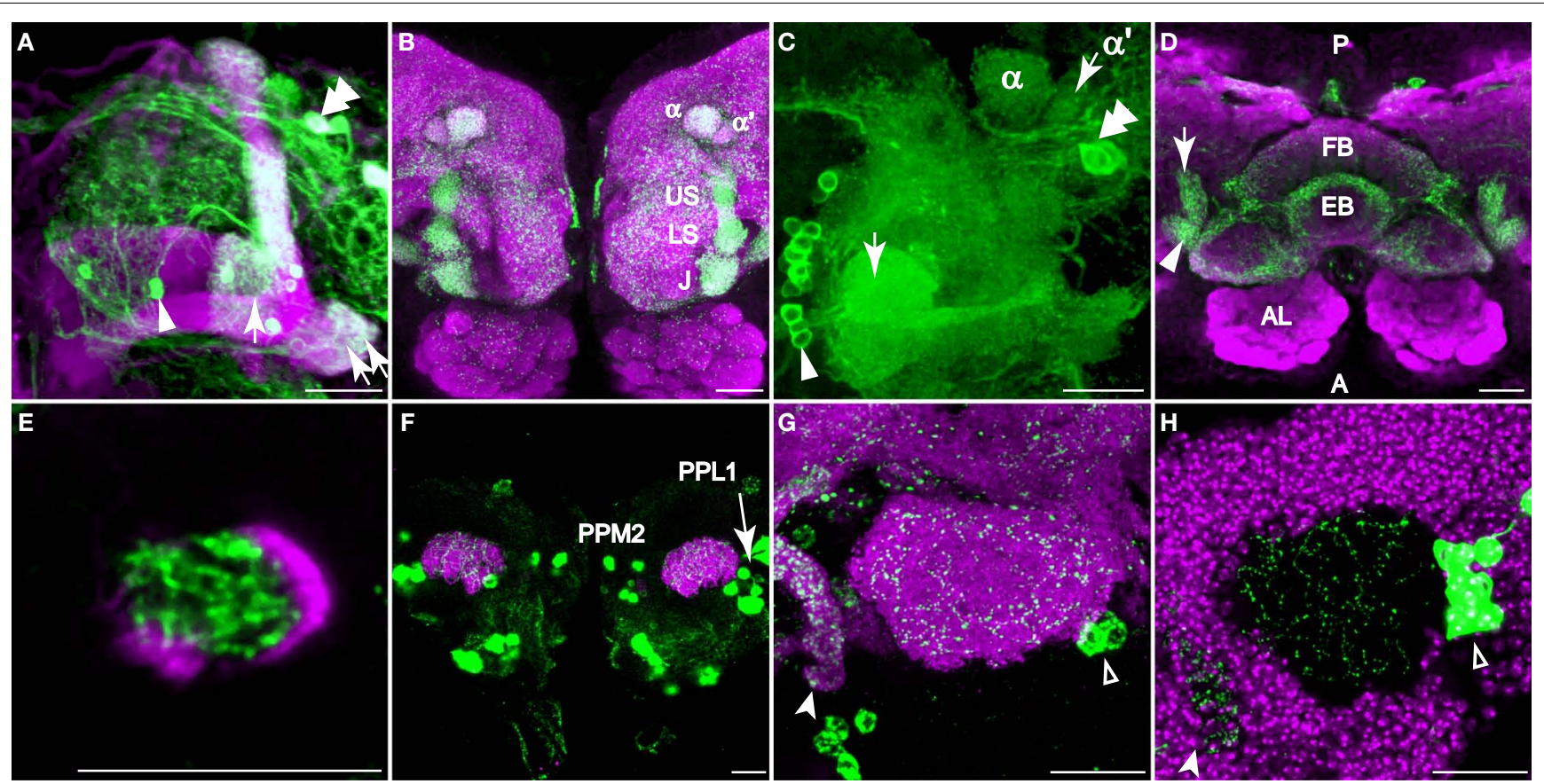

FIGURE 3 | Dopaminergic innervation of the mushroom bodies. Scale bar in all panels: $20 \mu \mathrm{m}$. (A-C) Innervation of the lobes as viewed from the anterior. (A) TH-GAL4 driven GFP expression in the vertical lobes, the junction (arrow), and the heel (double arrows). Note the sharp vertical boundary of green fluorescence just medial to the junction in the horizontal lobes, giving the impression that dopaminergic innvervation excludes much of the horizontal lobes. Magenta, antiFasll. Arrowhead, a PAM neuron. Double arrowhead, PAL neurons. Medial is to the left; lateral to the right. (B) TH-GAL4 driven UAS-syt.eGFP, showing dense synaptic puncta (green) within several zones of innervation of the vertical lobes, including the junction (J), the lower stalk (LS), the upper stalk (US), the tip of the $\alpha^{\prime}$ lobe $\left(\alpha^{\prime}\right)$ and the tip of the $\alpha$ lobe $(\alpha)$. Magenta, anti-nc82. (C) Robust anti-TH immunostaining of the medial tips of the horizontal lobes (arrow). Medial is to the left; lateral to the right. Arrowhead, PAM neurons. Double arrowhead, PAL neurons. (D) Dorsal view of dopaminergic innervation of the distal peduncle.
Green, TH-GAL4 driving UAS-syt.eGFP (green) showing dense puncta in the distal peduncle (arrow), heel (arrowhead) and the central complex (fan-shaped body, FB; ellipsoid body, EB). Magenta, anti-nc82. Posterior (P) is up; anterior $(\mathrm{A})$ is down. (E) Anti-TH signal (green) was observed in the distal peduncle, outlined by c305a-GAL4>UAS-mCD8::GFP (magenta). Anterior view. Medial is to the left; lateral to the right. (F) TH-GAL4>UAS-mCD8::GFP expression (green) in the calyx from a posterior perspective. Magenta, anti-nc82. Cell bodies of TH-GAL4 positive neurons (PPL1 and PPM2) in the posterior cortex can be seen. (G) TH-GAL4 driven UAS-syt.eGFP expression (green) in the calyx from a posterior viewpoint. Arrowhead, protocerebral bridge. Open arrowhead, PPL1 neurons. Magenta, anti-nc82. (H) Anti-TH signal (green) in the calyx. The calyx is shown as the void space surrounded by the densely packed mushroom body cell bodies (magenta, TOTO-3 nuclear stain). Arrow head, protocerebral bridge. Open arrowhead, PPL1 neurons. 
in the mushroom body heel (Figures 3A,D) and distal peduncle (Figure 3D). Interestingly, the $T H$-GAL4 expression in the vertical lobe appeared to be segmented into zones. Figure $3 \mathbf{B}$ illustrates this innervation of five such zones: the tip of the $\alpha$ lobe, the tip of the $\alpha^{\prime}$ lobe, the upper stalk, the lower stalk, and the area that joins the vertical and horizontal lobes (the junction).

The areas labeled by TH-GAL4 also expressed anti-tyrosine hydroxylase immunoreactivity (Figures 3C,E and 4A). In contrast to $\mathrm{TH}$-GAL4 expression however, anti-TH immunoreactivity was more widespread in the horizontal lobes and particularly intense at their distal tips (Figures 3C and 4A-D). Using NP1131-GAL4, $c 739-G A L 4$, and $c 305 a-G A L 4$ as markers of $\gamma, \beta$ and $\beta^{\prime}$ lobes, respectively, we localized putative DA processes to all three branches of the horizontal lobes (Figures 4A-D). These processes also appeared to form distinct zones. For instance, in addition to the junction region of the $\gamma$ lobe, three zones, $\gamma 3, \gamma 4$, and $\gamma 5$ could be distinguished
A

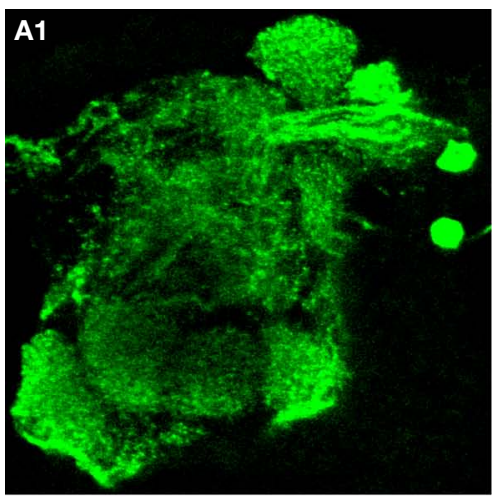

B

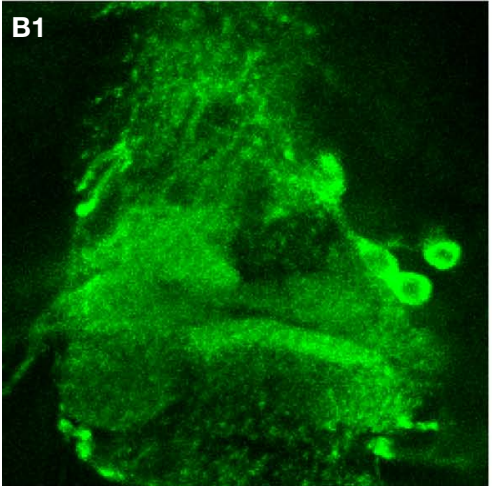

C

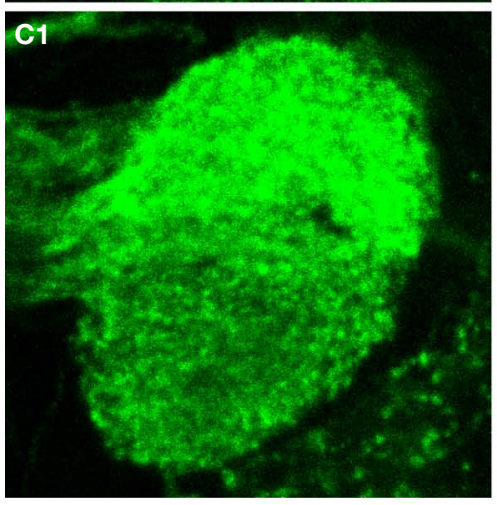

A2

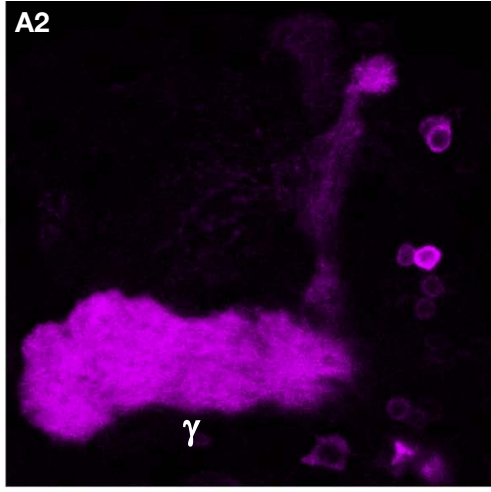

B2

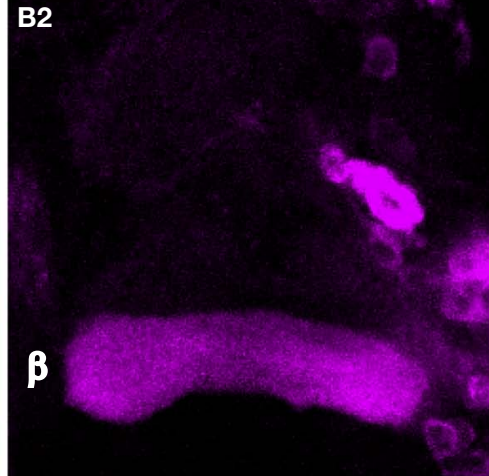

C2

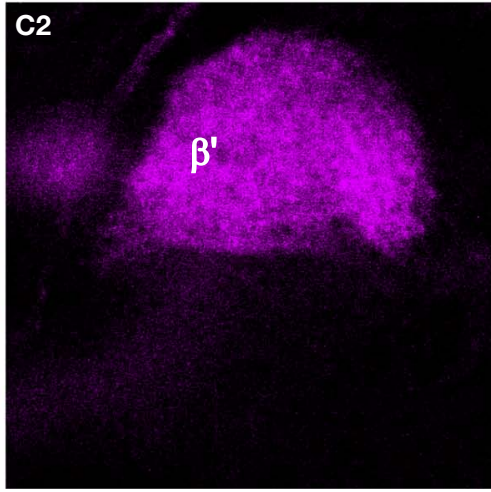

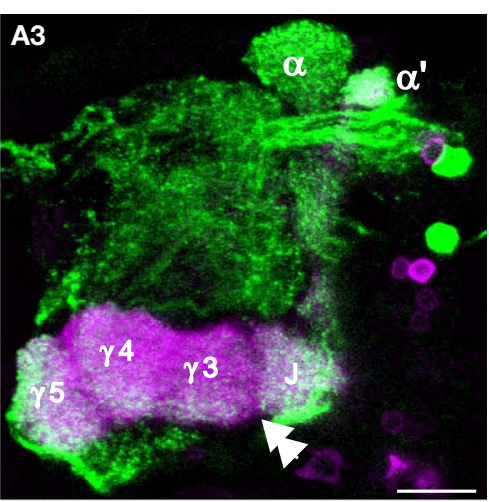

B3

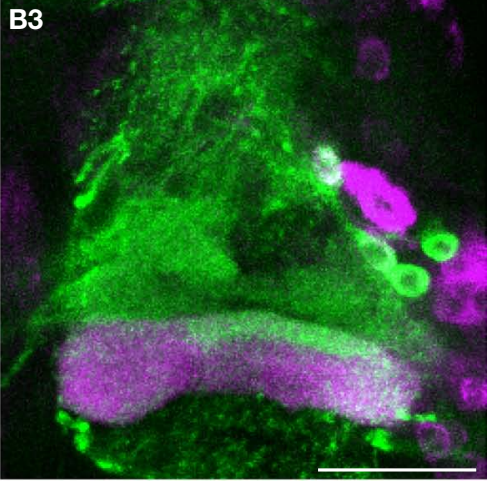

C3

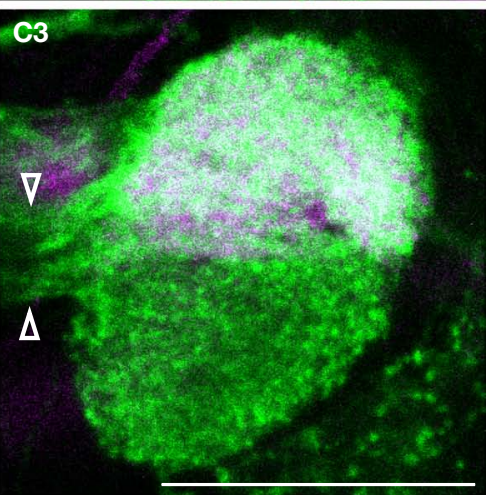

FIGURE 4 | Dopaminergic innervation of the horizontal lobes of the mushroom bodies. (A-D) Anti-TH immunostaining in the horizontal lobes. Maxiumum projection images of one hemisphere of frontal sections near the $\gamma, \beta^{\prime}$ or $\beta$ lobe. Medial is to the left; lateral to the right. Scale bar in all panels: $20 \mu \mathrm{m}$. (A1) Anti-TH immunostaining (green) of a brain from NP1131-GAL4>UAS-mCD8::GFP flies. (A2) Anti-GFP immunostaining (magenta) of the same brain. (A3) Merge of (A1) and (A2). Note the discontinuous signal (double arrowhead) between the junction $(\mathrm{J})$, and the medial segments of the $\gamma$ lobe. This discontinuity in signal is also apparent in panel (A1). (B1) Anti-TH immunostaining (green) of a brain from
c739-GAL4>UAS-mCD8::GFP flies. (B2) Anti-GFP immunostaining (magenta) of the same brain. (B3) Merge of (B1) and (B2). Rather widespread innervation of the $\beta$ lobe by dopaminergic processes was observed, with a strong horizontal bar of signal across the dorsal aspect of the $\beta$ lobe. (C1) Anti-TH immunostaining (green) of a brain from

c305a-GAL4>UAS-mCD8::GFP flies. (C2) Anti-GFP immunostaining (magenta) of the same brain. (C3) Merge of (C1) and (C2). Note the robust innervation of the medial tip of the $\beta^{\prime}$ lobe by dopaminergic processes. The open arrowheads point to the fibers connecting the ipsi- and contralateral tips of the medial lobes. (continued) 

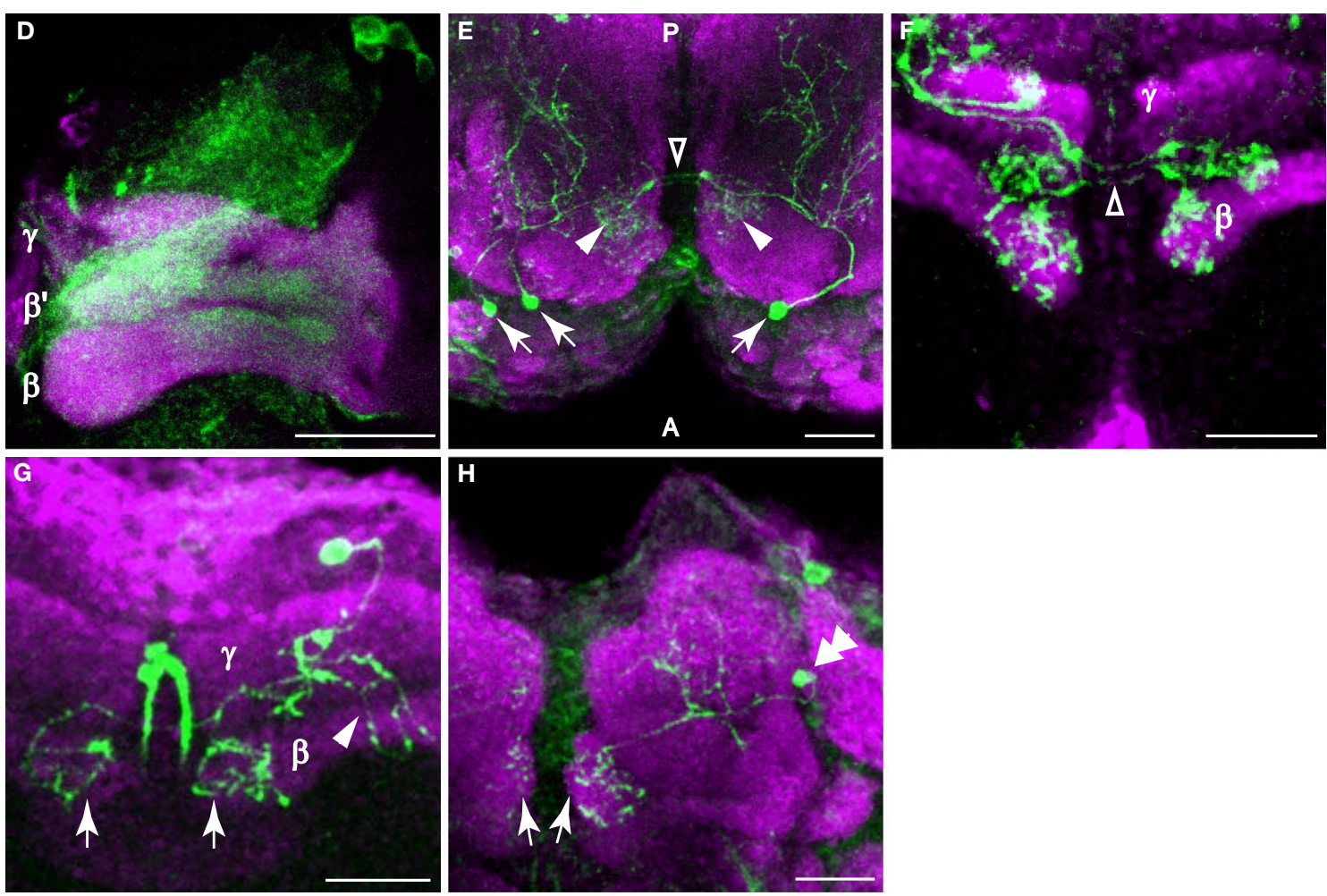

FIGURE 4 | Continued. (D) Anti-TH (green) and anti-GFP immunostaining (magenta) of a brain from OK107-GAL4>UAS-mCD8::GFPflies. (E) Dorsal view of a sample containing three flip-out PAM neurons (arrows) showing that PAM neurons innervate the horizontal lobes. These neurons extend processes posteriorly to the horizontal lobes (arrowheads) and to more posterior areas of the brain. The open arrowhead points to the fibers connecting the ipsi- and contralateral tips of the horizontal lobes. Green, TH-GAL4 flip-out GFP. Magenta, anti-nc82. Posterior $(\mathrm{P})$ is up; anterior $(\mathrm{A})$ is down. (F) Frontal section of a sample containing three flip-out PAM neurons. Only the processes at the tips of the horizontal lobes are shown in this image. The open arrowheads point to the fibers connecting the ipsi- and contralateral tips of the horizontal lobes. Green, TH-GAL4 flip-out GFP. Magenta, anti-FasII. (G) Ventral-anterior view of a flip-out PAM neuron that innervates the tips of the $\beta$ lobes (arrows). The arrowhead points to the processes that extend to more posterior areas of the brain. Green, TH-GAL4 flip-out GFP. Magenta, anti-Fasll. (H) Anterior view of a flip-out PAM neuron (double arrowhead) that innervates the tips of the $\gamma$ lobes (arrows). Green, TH-GAL4 flip-out GFP. Magenta, anti-nc82.
(Figure 4A3). There were also strong horizontal zones of expression such as the strong bar of signal across the dorsal aspect of the $\beta$ lobe (Figure 4B).

The calyx consists of the dendrites of mushroom body neurons and receives odor information from projection neurons. In contrast to prior studies, we observed putative DA terminals in the calyces. Using UAS-mCD8:GFP as a reporter for TH-GAL4, we observed a sparse network of processes in the calyx (Figure 3F). Synaptic puncta were observed in the calyx with synaptotagmin.eGFP as a reporter (Figure 3G) and anti-TH immunoreactivity was visible as fine dots on the network of DA processes (Figure 3H).

\section{THE PAM CLUSTER INNERVATES THE HORIZONTAL LOBES OF THE MUSHROOM BODIES}

To identify the source of the innervation to the mushroom bodies, we used TH-GAL4 to drive a UAS $>C D 2, y^{+}>C D 8:: G F P$ transgene combined with an $\mathrm{Hs}$-flp transgene (Wong et al., 2002). Animals bearing these transgenes were treated with mild heat-shock at various developmental stages, which induced the excision of the $C D 2$, $y^{+}$cassette and the expression of the CD8::GFP tag in a random subpopulation of TH-GAL4 expressing neurons. We were then able to visualize the projection patterns of one or a few clones without interference from other TH-GAL4 expressing neurons. Each of the projection patterns described below, except those shown in Figures 4G,H, has been observed in more than one sample (Table 2). Three clusters of putative DA neurons, PAM, PPL1 and PPL2ab, were found to innervate the mushroom body neuropil (see Figures 2D,G-I,J-L for TH-immunoreactivity of these clusters).

The PAM cluster of small neurons was located in the most anterior part of the protocerebrum (Figures 1B,C). These neurons projected bilaterally to the distal tips of the horizontal lobes (Figures 4E-H). Other branches of these neurons arborized in the mushroom body satellite area and also extended posteriorally (Figures 4E,G). We did not obtain PAM clones that innervate other areas of the horizontal lobes. One possible reason is that our flip-out study did not saturate all TH-GAL4 expressing neurons (see Table 2). A second possibility is that some PAM neurons were not represented by the TH-GAL4 line and therefore remained hidden. A third possibility is that some sub-areas may be innervated by uncharacterized DA neurons residing outside of the protocerebrum. 
Table 2 | Classes of TH-GAL4 flip-out clones that project to the mushroom body.

\begin{tabular}{|c|c|c|c|c|}
\hline Type & Cluster & Mushroom body area innervated & Number of samples observed ${ }^{a}$ & Heat shock time \\
\hline 1 & PAM & Medial portion of horizontal lobes ${ }^{b}$ & 11 & Pupal \\
\hline 2 & PPL1 & $\alpha \operatorname{Tip}$ & 11 & Larval and pupal \\
\hline 4 & PPL1 & Upper stalk & 5 & Pupal \\
\hline 5 & PPL1 & Lower stalk and junction & 13 & Larval and pupal \\
\hline 8 & PPL2ab & Calyx and entire lateral horn & 2 & Pupal or late larval \\
\hline
\end{tabular}

a Some samples of the PAM and PPL 1 clusters contained multiple clones.

${ }^{b}$ Different subtypes have been observed, including single clones projecting to the tips of the $\gamma$ lobes, the tips of the $\beta$ lobes, or areas roughly corresponding to the tips of the $\beta^{\prime}$ lobes. Due to the frequent occurrence of multiple clones and relatively low fluorescence of single cells, we do not have an $n \geq 2$ of high quality single clones to confidently identify all subtypes.

\section{THE PPL1 CLUSTER INNERVATES THE VERTICAL LOBES OF THE MUSHROOM BODIES}

The PPL1 cluster of 12 or so neurons resides laterally to the calyx in the posterior brain and extends projections towards the dorsal and anterior. Despite the proximity to the calyx, close examination failed to reveal processes that entered the calyx. Within this cluster, we observed four subtypes of neurons that projected to one specific subarea of the vertical lobes of the mushroom bodies: the tip of $\alpha$ lobe (Figures 5A-C), the tip of $\alpha^{\prime}$ lobe (Figure 5D), the upper stalk (Figure 5E), and the lower stalk and junction area (Figures 5F, G; Table 2). An additional subtype of PPL1 neurons projected to the heel and distal peduncle (Figures $5 \mathbf{H}-\mathbf{J}$ ). Neurons of the latter three subtypes have been identified as mushroom body extrinsic neurons MB-V1, MB-MV1, and MBMP1, respectively (Tanaka et al., 2008). The cell bodies of some TH-GAL4 positive neurons exhibit short processes in addition to the primary neurite (Figure 5K). We also observed three subtypes of neurons that do not project to the lobes, but to areas surrounding the lobes. One subtype of PPL1 neuron arborized around the edge of the medial portions of the horizontal lobes (Figures 5L,M). A second subtype innervated broad areas surrounding the ipsilateral vertical lobes (not shown), especially in areas medial to the lobes (not shown). A third subtype gave rise to one branch that arborized in areas posterior to the ipsilateral vertical lobes and another branch that innervated the entire span of the superior arch (Figures $\mathbf{5 N}, \mathbf{O}$ ).

Most PPL1 neuron clones we obtained targeted bilateral, symmetrical destinations while extending additional, complex arborizations in the ipsilateral hemisphere (Figures $\mathbf{5 C , G}, \mathbf{J}$ ). These additional arborizations were characteristic of each subtype, just as the bilateral targets were characteristic of each subtype of PPL1 neuron, suggesting that through PPL1 neurons, subareas of mushroom bodies are linked stereotypically to distinct brain regions. It is possible that additional subtypes of PPL1 neurons exist but were not detected. On the other hand, it remains possible that some subtypes of flip-out clones may not be authentic DA neurons. The existence of eight distinct projection patterns from the PPL1 cluster that contains $\sim 12$ neurons suggest that there is probably only 1-2 neurons in each cluster targeted to each distinct neuropil region.

\section{THE PPL2ab CLUSTER INNERVATES THE CALYX OF THE MUSHROOM BODIES}

The cell bodies of PPL2ab neurons were located on the lateral edge of the protocerebrum near the optic lobes. We observed two subtypes of PPL2ab neurons that targeted the ipsilateral calyx and both also invaded the lateral horn, another higher-order center along with the mushroom bodies for olfactory information processing in the fly brain (Table 2). One type of PPL2ab neuron extended its processes to the calyx, the inferior portion of the lateral horn, and broad areas in the middle superior medial protocerebrum (Figures 6A,B). This type of neuron was identified as the MB-C1 extrinsic neuron in Tanaka et al. (2008). Another type of PPL2ab neuron innervated the calyx, the entire lateral horn, and broad areas of the middle superior protocerebrum (Figures 6C,D). In addition to these two subtypes of neurons, the PPL2ab group also contained at least two other subtypes of neurons that did not project to the calyx. One subtype innervated the lobula, in addition to broad protocerebral areas (Figure 6E). Another subtype innervated the posterior lateral protocerebrum (Figure 6F). As all PPL2ab neurons exhibit coincidental expression of tyrosine hydroxylase (Figures $\mathbf{2 J}-\mathbf{L}$ ), it is likely that their innervation of the calyx is DA. The above data showed that at least four different subtypes of neurons exist in the PPL2ab cluster.

Nässel and Elekes (1992) identified in the blow fly two subclusters of PPL2ab neurons: the "a" group consists of six more dorsally located neurons and the "b" group consists of two slightly larger and more ventrally located neurons. They suggested that there might be homologous counterparts in Drosophila. We did not identify two such subclusters although they may exist.

We also obtained a third type of TH-GAL4 flip-out clone that innervated the ipsilateral calyx (Figure 3 in Supplementary Material). The cell body is located ventrolateral to the calyx (Figures 3A-D,F in Supplementary Material). Its primary process extended dorsally and anteriorly to reach the peduncle. There, one branch turned posteriorly along the periphery of the peduncle and spread throughout the entire calyx (Figures 3B,F in Supplementary Material). Another branch extended anteriorly to to the vincinity of the horizontal lobes (Figures 3A,B,G in Supplementary Material). This type of neuron resembles the MB-CP1 extrinsic 

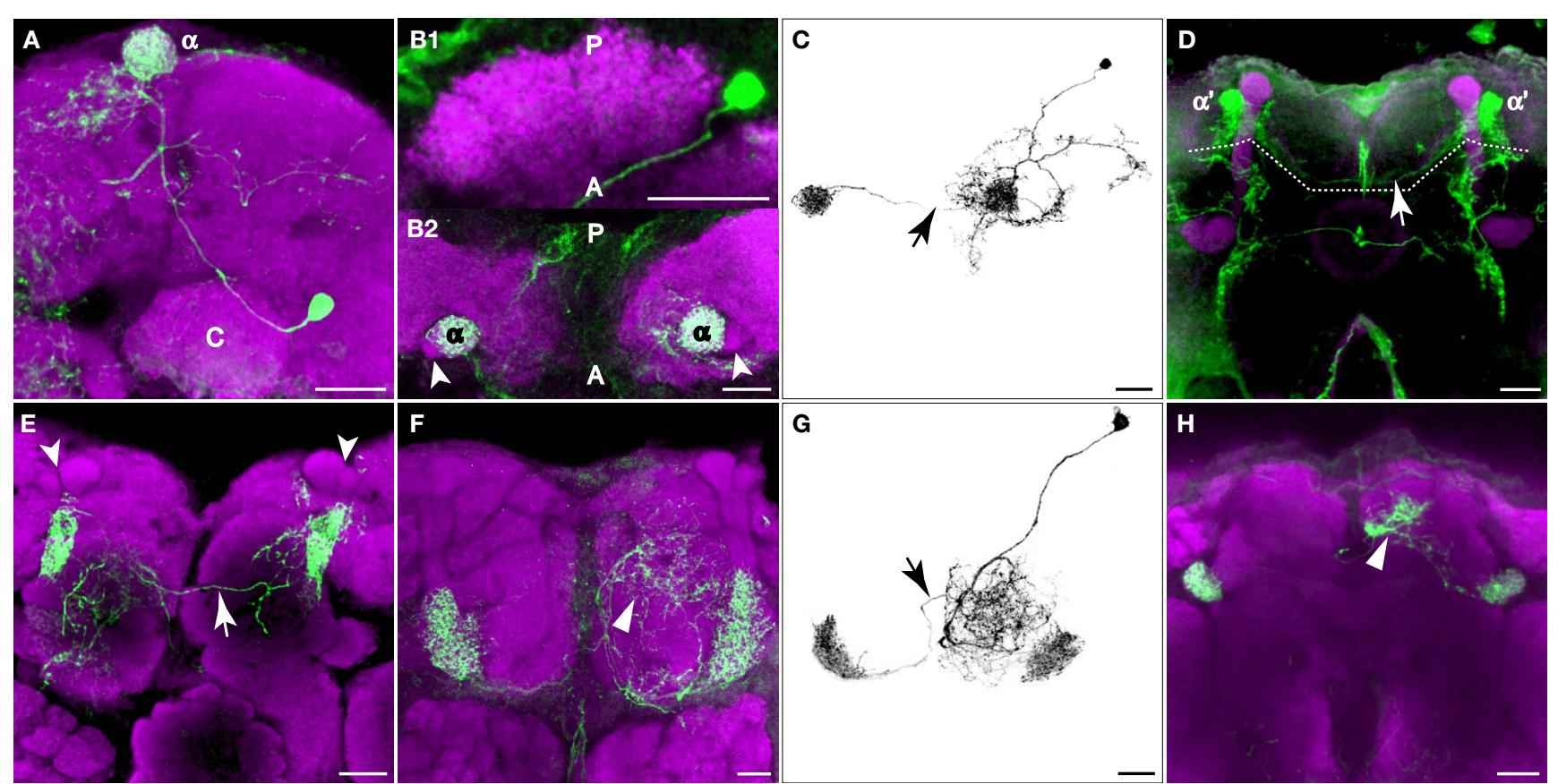

FIGURE 5 | PPL1 neurons innervate distinct areas of the mushroom body lobes. Green, anti-GFP from TH-GAL4 flip-out clones. Magenta, anti-Fasll immunostaining in panel (D), anti-nc82 immunostaining in all others. Grayscale, GFP signal with background removed along with other unrelated structures. Scale bar in all panels: $20 \mu \mathrm{m}$. (A) One type of PPL1 neuron innervates the tip of the $\alpha$ lobe. Posterior view. Medial is to the left; lateral to the right. The cell body is located lateral to the calyx (C). (B1,B2) Dorsal view of another PPL1 neuron. Posterior (P) is up; anterior (A) is down. (B1) Cell body is located lateral to the calyx. The primary process runs along the anterior margin of the calyx rather than entering it. There was no indication at this level of resolution that the calyx is innervated by PPL1 neurons. (B2) The tips of the $\alpha$ lobes $(\alpha)$, but not the $\alpha^{\prime}$ lobes (arrowheads), are innervated bilaterally by the PPL1 neuron cell body shown in (B1). (C) The entire track of the neuron in panels (B1) and (B2) viewed from a dorsal perspective. Arrow points to the fiber providing innervation to the contralateral $\alpha$ lobe tip. There is considerable branching of the neuron in the dorsal ipsilateral protocerebrum prior to contacting the ipsilateral $\alpha$ lobe. (D) One type of PPL1 neuron bilaterally innervates the tip of $\alpha^{\prime}$ lobe. Robust innervation of the tip of the $\alpha^{\prime}$ lobe is observable in this PPL1 clone. The arrow points to the fiber connecting the two hemispheres. Only the processes innervating the $\alpha^{\prime}$ lobe tip are from the PPL1 neuron clone (above dotted line); other labeled processes are from PPM3 clones in this brain that innervate the fan-shaped body (below dotted line). The cell body for the PPL1 neuron is not visible in this image stack that represents an anterior region of the brain. (E) One type of PPL1 neuron bilaterally innervates the upper stalk of the vertical lobes. The upper stalk is the columnar region of the vertical lobes that is ventral to the tips of the $\alpha$ and $\alpha^{\prime}$ lobes (arrowheads). The arrow points to the fiber connecting the two hemispheres. All of the fluorescent processes in the right hemisphere from this viewpoint as well as the fiber link is from the clone bilaterally innervating the upper stalks of the vertical lobes. The fluorescent processes in the left hemisphere ventromedial to the left upper stalk is from another clone in the brain. (F) One type of PPL1 neuron innervates the lower stalk and junction. View from the anterior. Arrowhead points to processes in the anterior inferior medial protocerebrum from the same PPL1 neuron that innervates the junction and lower stalk. (G) Dorsal view of another PPL1 neuron that bilaterally innervates the junction and lower stalk. The PPL1 neuron ramifies broadly throughout the anterior inferior medial protocerebrum en route to the junction and lower stalk. Arrow points to the fiber providing innervation to the contralateral junction and lower stalk. (H) One type of PPL1 neuron bilaterally innervates the heel and distal peduncle. In this projection image from an anterior perspective, the heel obscures the distal peduncle. Arrowhead, processes from the PPL1 neuron innervating the heel, exhibiting broad ramification in the superior medial protocerebrum prior to contacting the ipsilateral heel/distal peduncle. (continued) neuron identified in Tanaka et al. (2008), but it did not reliably express $T H-G A L 4$ and was not observed in population samples (Figure 1D), nor in anti-TH staining (Figure 2A). A somewhat analogous situation concerns the PPM4 neuron, which was observed only very rarely in population samples but was present multiple times in flip-out clones (Figure 1F in Supplementary Material).

\section{INNERVATION OF THE CENTRAL COMPLEX}

In addition to the mushroom bodies, another neuropil, the central complex, is also heavily innervated by DA terminals (Figure 3D). The central complex of the Drosophila is involved in controlling locomotion (Strauss, 2002) and may also be involved in learning and memory (Liu et al., 2006). It consists of the ellipsoid body, the fan shaped body and superior arch, the noduli, and the protocerebral bridge. In the course of our clonal analysis to identify mushroom body-targeting neurons, we came across many TH-GAL4 flip-out clones that innervated various structures of the central complex. Three clusters of putative DA neurons were found to be the source of projection to these structures.

A major source of innervation to the central complex was the PPM3 cluster. One subtype of PPM3 neuron projected to the fan shaped body (Figures 2I,J in Supplementary Material). It also had one branch that extended dorsally from the corner between the vertical and horizontal lobes, parallel to the vertical lobes (Figure 5D; Figure 2J in Supplementary Material). Another subtype of PPM3 neuron projected to the fan shaped body and the paired noduli. This type of neuron had a branch that projected towards the anterior 


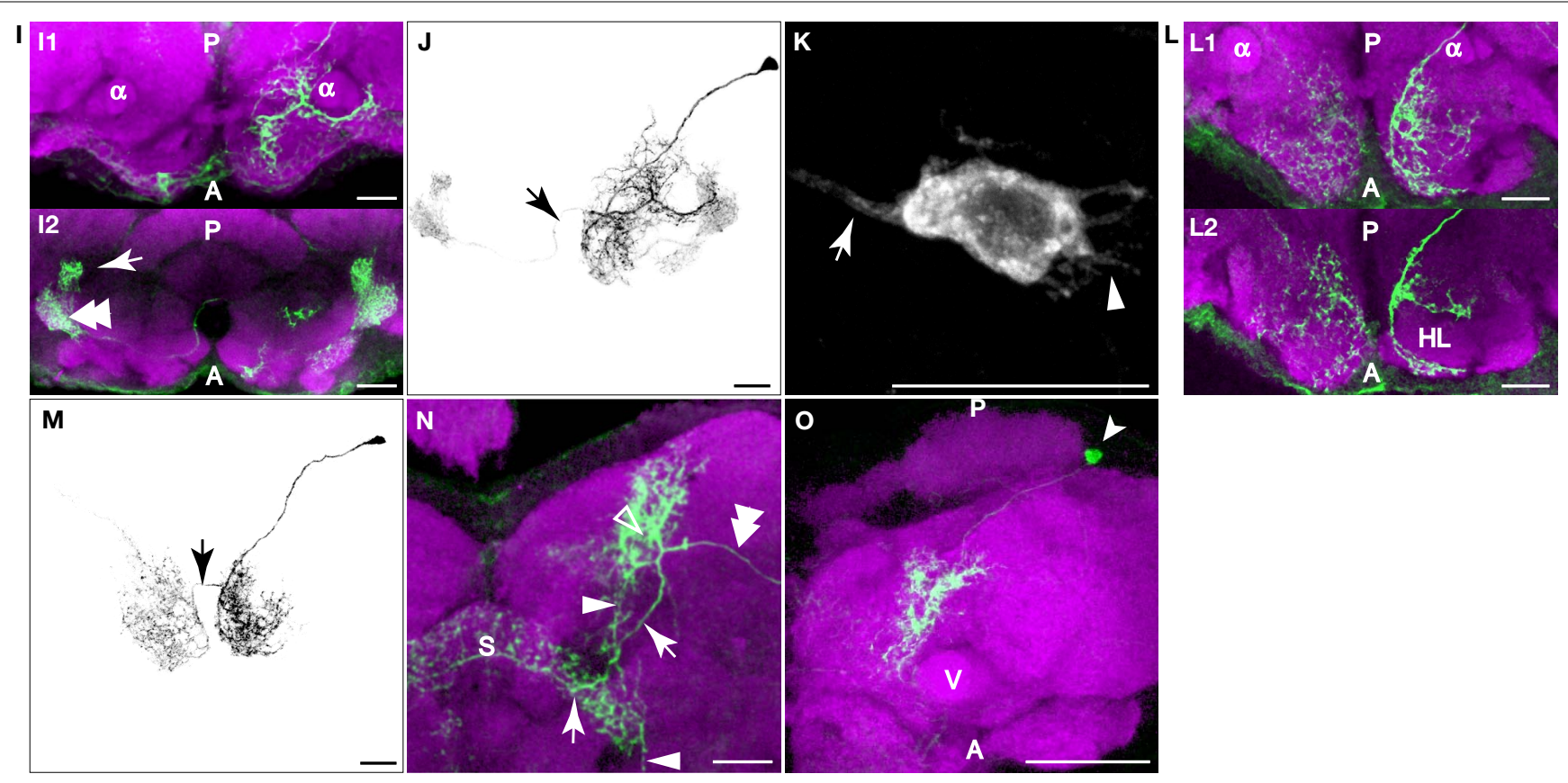

FIGURE 5 | PPL1 neurons innervate distinct areas of the mushroom body lobes. (I) Dorsal view at two levels of another PPL1 neuron innervating the heel. Panel (I1) shows the neurons at the dorsal/ventral level of the tips of the vertical lobes and panel (12) shows the neuron at the level of the heel. Posterior (P) is up; anterior $(\mathrm{A})$ is down. Panel (I1) shows that the more dorsal processes of this neuron ramify around the anterior surface of the vertical lobes before innervating the heel (double arrowhead in (12)) and the distal peduncle (arrow in (I2)). (J) Entire track of the neuron in panel (I). The neuron extends a process that branches extensively around the ipsilateral vertical lobe in the anterior protocerebrum, prior to innervating the ipsilateral heel and the distal peduncle. Arrow points to the fiber providing innervation to the contralateral heel and peduncle. (K) Cell body of the PPL1 neuron shown in panel (F). The arrow points to the primary neurite that projects to the mushroom bodies. The arrowhead points to short processes. (L) One type of PPL1 neuron innervates the anterior superior and inferior medial protocerebrum. (L1) Image from a dorsal perspective taken at a level near the tips of the vertical lobes showing broad innervation of the anterior superior medial protocerebrum. (L2) An image from a deeper plane showing the PPL1 neuron branches that surround the horizontal lobes $(\mathrm{HL})$ in the anterior inferior medial protocerebrum. This is more obvious in the right hemisphere as viewed because of the tilt of the specimen. (M) Depiction of the neuron shown in panel (L). The arrow points to the fiber providing for bilateral innervation of the anterior superior and inferior medial protocerebrum. (N) One type of PPL1 neuron extends a process (double arrowhead) to a region posterior to the tips of the vertical lobes (open arrowhead). One secondary branch (arrow) innervates the superior arch (S). Another branch (arrowhead) extends ventrally to a region medial to the vertical lobes and posterior to the horizontal lobes. (0) Dorsal view of another PPL1 neuron that has the same innervation pattern as the one shown in panel (N). Arrow head points to the cell body lateral to the calyx. There is dense innervation of the region posterior to the vertical lobes $(\mathrm{V})$. Only the most dorsal portion of the brain is shown here. superior medial protocerebrum and around the mushroom body lobes (Figures 2K-M in Supplementary Material). Since both of these subtypes of neurons had branches that are in the proximity of the mushroom bodies, it is possible that the neurons may link the central complex and the mushroom body lobes for some function. A third subtype of PPM3 neuron projected to the ellipsoid body (Figure $2 \mathrm{~N}$ in Supplementary Material). As described above, the superior arch was innervated by one subtype of PPL1 neuron that also projected to areas posterior to the vertical lobes (Figures 5N,O). The protocerebral bridge was found to be innervated by a pair of neurons located in the tritocerebrum (Figures 2O,P in Supplementary Material), designated T1 by Nässel and Elekes (1992).

\section{RESPONSES OF DA PROCESSES IN THE MUSHROOM BODY LOBES TO ELECTRIC SHOCK AND ODORS}

In Drosophila, it has been shown that synaptic transmission from DA neurons is required for robust olfactory conditioning using electric shock as a negative reinforcer (Schwaerzel et al., 2003). To further understand the role of the DA neurons for learning at a cellular level, we examined the responses of the DA processes to odor and the electric shock stimuli used for olfactory classical conditioning. We used TH-GAL4 to drive UAS-G-CaMP, a calcium indicator, to visualize putative DA neuron activity. By imaging from the dorsal perspective of the fly brain and taking advantage of the optical sectioning capability of the confocal microscope, we were able to record the activity of putative DA processes at various depths corresponding to different segments along the vertical lobes. We imaged the tips of $\alpha$ and $\alpha^{\prime}$ lobes, the upper stalk region, and the lower stalk/junction region, each shown above to be innervated by a distinct subtype of PPL1 neuron. The location of all four areas is illustrated in Figure 3B from a frontal perspective, although the functional imaging was performed from a dorsal perspective in which the tips of $\alpha$ and $\alpha^{\prime}$ lobes can be captured simultaneously (Figure 7A). The upper stalk and the lower stalk/junction regions were captured by imaging at different 

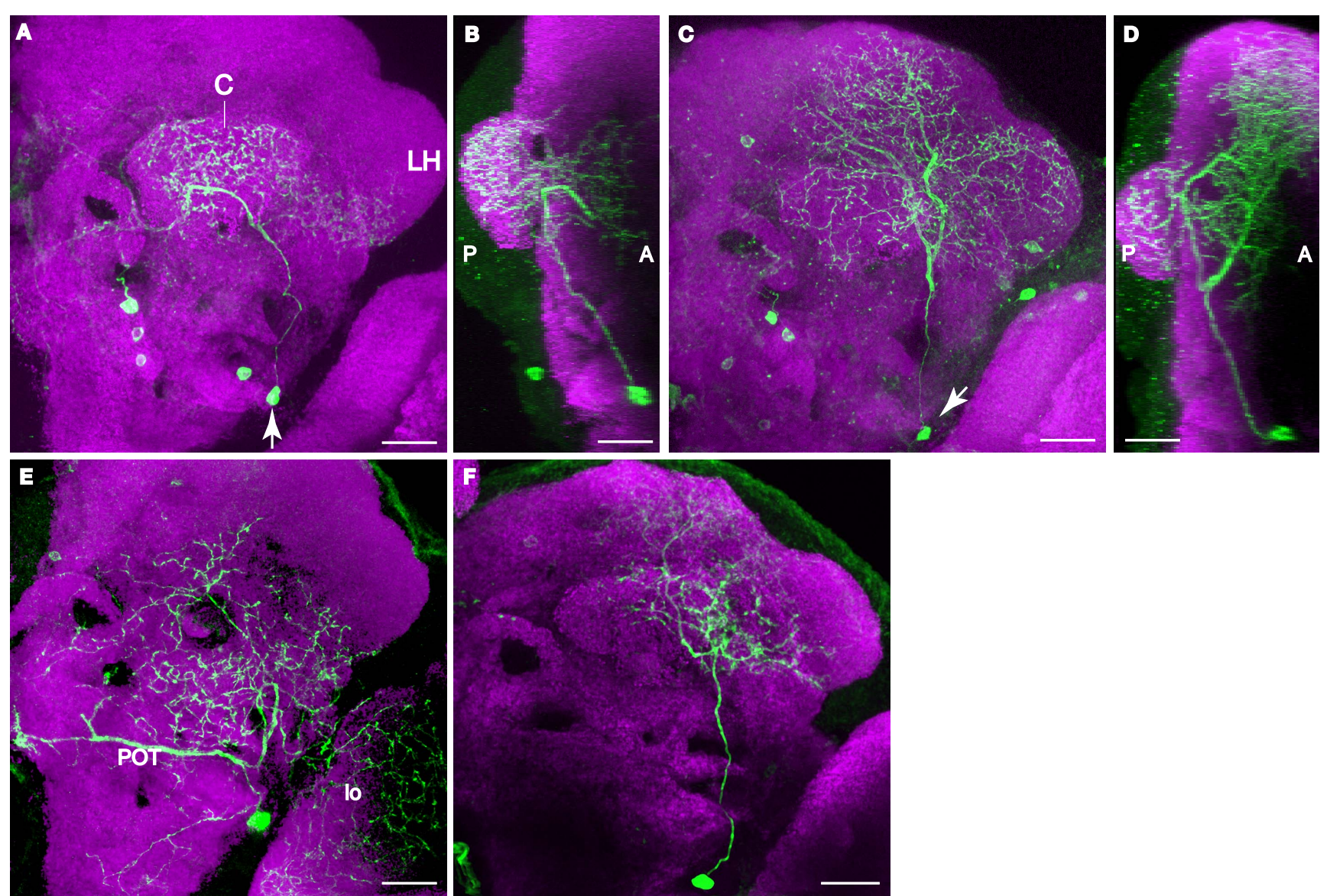

\section{FIGURE 6 |Two types of PPL2ab neuron innervate the calyx of the}

mushroom body. In contrast to the PPL1 neurons that bilaterally innervate the mushroom body lobes, the innervation of each calyx is unilateral. Scale bar in all panels: $20 \mu \mathrm{m}$. (A) Posterior view of one type of PPL2ab neuron. The arrow points to the cell body. The primary neurite from this neuron extends dorsally. One branch innervates the calyx $(\mathrm{C})$. A second branch innervates the adjacent posterior inferior lateral protocerebrum and the ventral portion of the lateral horn (LH). A third branch projects medially and innervates the middle inferior medial protocerebrum. Medial is to the left, lateral to the right. (B) Projection image of the neuron in panel (A) from a lateral point of view. Anterior is to the right, posterior to the left. (C) Posterior view of aother type of PPL2ab neuron (arrow points to the cell body). The primary neurite from this neuron extends dorsally and ramifies to innervate the calyx, the entire lateral horn and the middle superior protocerebrum. Medial is to the left, lateral to the right. (D) Projection image of the neuron in panel (C) from a lateral point of view. Anterior is to the right, posterior to the left. (E) Posterior view of a PPL2ab neuron that does not project to the calyx. This neuron extends a dorso-medial branch that travels along the posterior optical track (POT) and ramifies near the dorsal end of esophagus in both hemispheres, a lateral branch that innervates the lobula (lo), and dorsal processes that innervates broad areas from the posterior lateral protocerebrum, the superior posterior slope, and the middle inferior protocerebrum. (F) Posterior view of another PPL2ab neuron that does not project to the calyx. It innervates the posterior inferior lateral protocerebrum and the posterior superior lateral protocerebrum. depths. To avoid potential complications produced by prior odor or shock exposure, each animal was used for a single stimulus presentation (odor or electric shock, Figure 7) or conditioning protocol (Figure 8) at a plane representing $\alpha \alpha^{\prime}$, the upper stalk or lower stalk/junction.

All four areas imaged responded to electric shock (Figure 7). However, there were significant differences in the amplitudes of the responses (Figure 7B, left panel). The lower stalk/junction area exhibited the strongest response; the amplitude was about tenfold higher than that of the weakly responding $\alpha^{\prime}$ tip or the upper stalk area. The $\alpha$ tip also responded strongly, although less than the lower stalk/junction area. The amplitude of the fluorescent response was proportional to the current delivered to the fly (Figure 7B, left panel).
Similarly, all four areas also responded to odor, with significant differences in amplitudes of responses (Figure 7B, middle panel). The $\alpha^{\prime}$ tip exhibited the greatest response to 4-methylcyclohexanol $(\mathrm{MCH})$, while the $\alpha$ tip exhibited the lowest response. The amplitude of response of the junction area was intermediate and significantly different from the responses of both the $\alpha^{\prime}$ and $\alpha$ tips. The responses in the upper stalk area was not significantly different from that of the $\alpha$ tip. We also imaged the response of DA processes in the upper stalk area to another odor, 3-octanol (OCT). The responses increased along with odor concentration (Figure 7B, right panel). Across stimulus modes, the lower stalk/junction and $\alpha$ tip areas responded more robustly to electric shock than to $\mathrm{MCH}$, while the $\alpha^{\prime}$ and upper stalk areas responded more robustly to $\mathrm{MCH}$ than to electric shock. The contrasting response properties of the $\alpha$ and $\alpha^{\prime}$ 
A
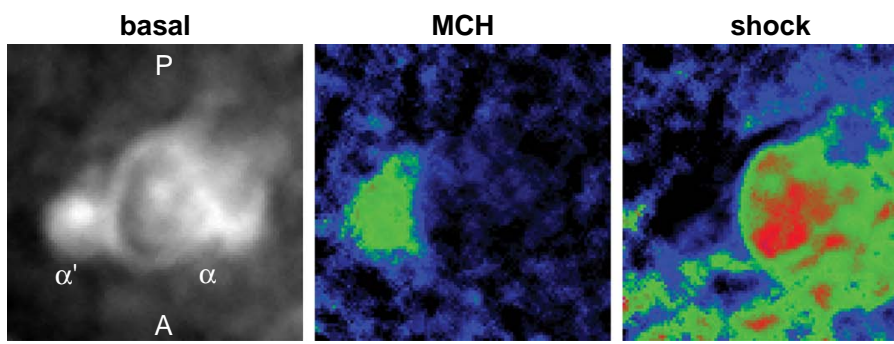

B 80

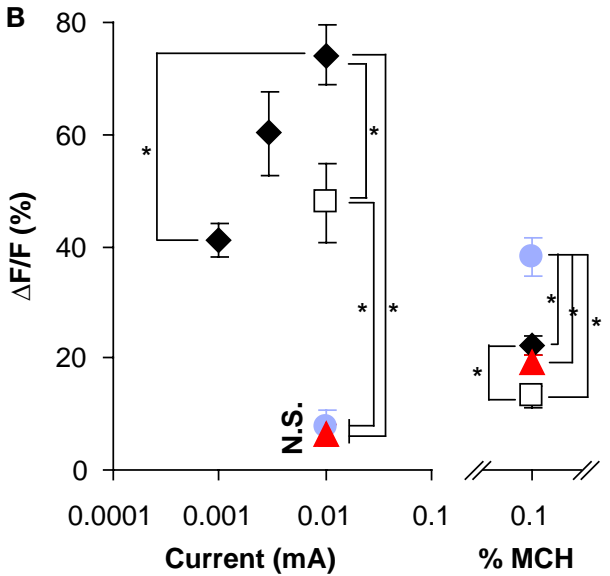

$\alpha$

$\alpha$

$\Delta$ upper stalk

lower stalk-junction
140

68

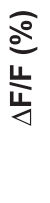

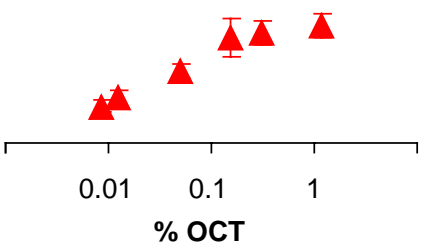

FIGURE 7 | Odor and shock response properties of dopaminergic processes in four subareas of the mushroom body lobes. (A) General response properties shown from one TH-GAL4>UAS-G-CaMP fly. Left, basal fluorescence. Middle, response to $0.1 \%$ methylcyclohexnol $(\mathrm{MCH})$ presented to the antennae of the fly. Right, Response to $0.01 \mathrm{~mA}$ electric shock presented to the legs. All three images are averages of five successive frames either before (basal) or at the peak responses ( $\mathrm{MCH}$ and shock). The tips of $\alpha$ lobe and $\alpha^{\prime}$ lobe were imaged simultaneously. $\mathrm{MCH}$ elicits a robust response in the PPL1 neuron processes that innervate the $\alpha^{\prime}$ tip and a much more modest response in those that innervate the $\alpha$ tip. Electric shock elicited a robust response in the processes that innervate the $\alpha$ tip and a much more modest response in those that innervate the $\alpha^{\prime}$ tip. PPL1 neurons therefore exhibit differential response properties to these stimuli. (B) Group data. (Left panel) At the same current level, the response to electric shock was significantly higher in lower stalk/ junction ( $n=12$ ) and $\alpha$ tip $(n=6)$ than in the $\alpha^{\prime}$ tip $(n=6)$ or the upper stalk $(n=12)$. The response increased in the lower stalk/junction with increasing current ( $n=12$ at each current level). (Middle panel) Response to $\mathrm{MCH}$ was significantly higher in the tip of $\alpha^{\prime}(n=31)$ than in the tip of $\alpha(n=31)$, the upper stalk ( $n=45)$, or the lower stalk/junction $(n=36)$. It was also significantly higher in the lower stalk/junction than in the $\alpha$ lobe tip. (Right panel) Responses in the upper stalk increased with increased odor concentration ( $n=7$ for each point). Data points represent mean \pm SEM. ${ }^{*}$ Indicates significant difference $(p \leq 0.0002)$. Each animal was subjected to only one stimulus at one dose to avoid complications due to prior exposure. tips for the same animal are illustrated in Figure 7A, wherein the $\alpha^{\prime}$ tip responded more robustly than the $\alpha$ tip to $\mathrm{MCH}$, but electric shock produced a more robust response in the $\alpha$ tip.

As observed in Figure 5, individual PPL1 neurons that innervate a specific subcompartment of the mushroom bodies also extend other processes that ramify outside of the mushroom bodies, especially in the ipsilateral hemisphere. For instance, processes medial to and anterior-lateral to the $\alpha$ tip in the left hemisphere were observed in single PPL1 neurons that innervates the tip of the $\alpha$ lobe (Figure 5B2, see also Figure 5C). We think that, among other possibilities, the increase of fluorescence in response to electric shock in areas medial to and anterior-lateral to the $\alpha$ tip in Figure 7A (right panel) may reflect synchronous activity of collaterals of the neurons that innervate the $\alpha$ tip. In population samples of TH-GAL4 expressing neurons, collaterals from the PPL1 neurons form extensive "clouds" of signal outside the mushroom body lobes, notably in the so-called satellite area between the vertical and horizontal lobes (Figures 3A,B), where a similar cloud of THIR signal was also detected (Figure 3C). We have also observed significant responses to electric shock in the satellite area while in the same image, the upper stalk area was hardly responsive (not shown).

\section{RESPONSES OF THE DA PROCESSES IN THE MUSHROOM BODY LOBES TO OLFACTORY CONDITIONING}

We further examined whether there was any change in the response properties of putative DA processes to an odor after the odor was paired with electric shock, and whether there were differences between the four subareas in their responses to such pairing. Five groups were assayed (Figure 8A): naïve, forward (1 min odor and 12 shock pulses, odor preceding shock by $5 \mathrm{~s}$ ), backward (1 min odor and 12 shock pulses, shock preceding odor by $45 \mathrm{~s}$ ), CS only ( 1 min odor), and US only (12 shock pulses only). In behavioral conditioning experiments, only forward pairing leads to associative memory (Tully and Quinn, 1985; Yu et al., 2006). Forward pairing of stimuli presented to an immobilized fly under the microscope has been shown to produce memory traces in projection neurons and dorsal paired medial (DPM) neurons at 3-7 and 30-60 min after training, respectively (Yu et al., 2004, 2005). Using a different 


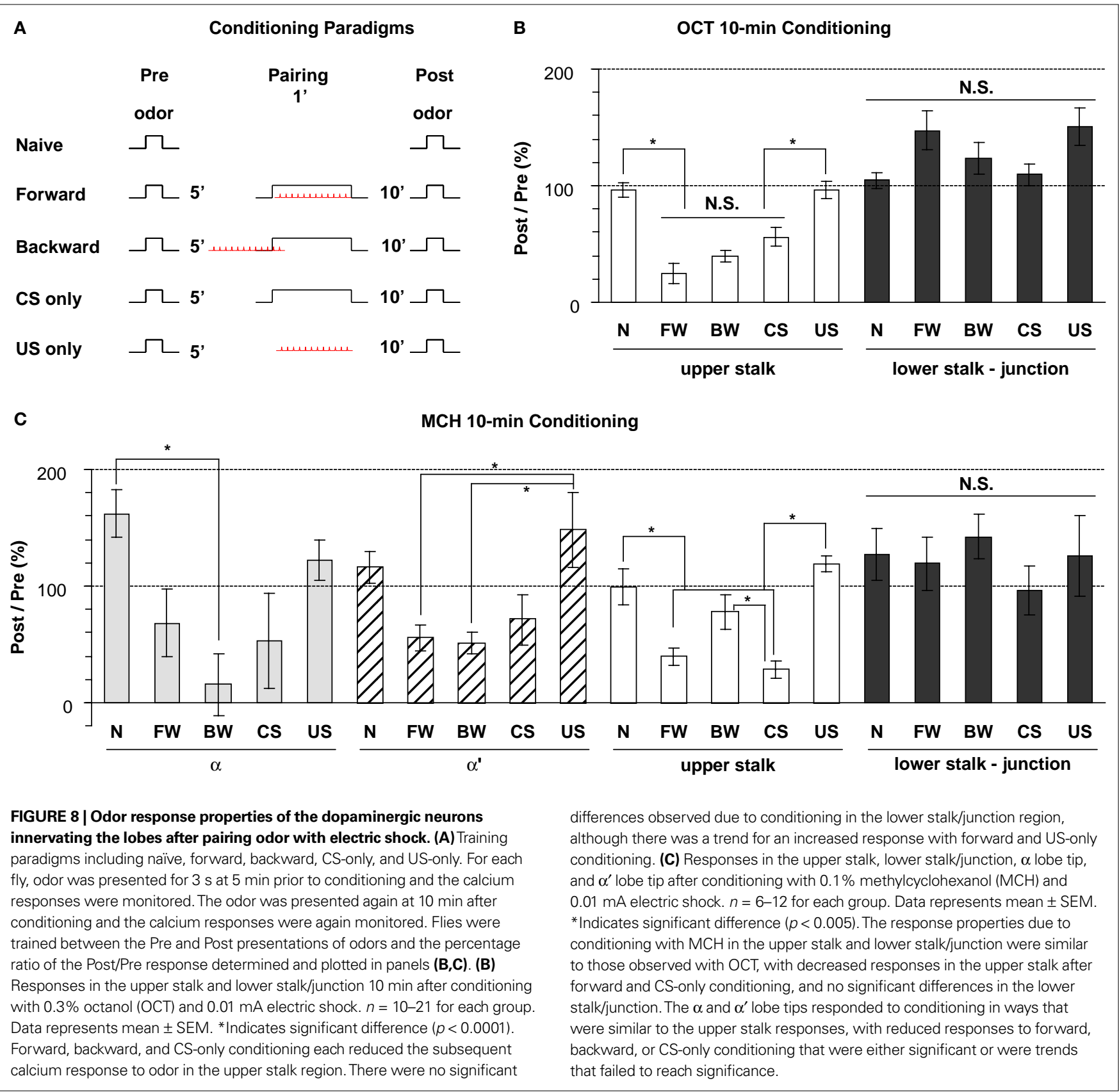

pairing protocol, Riemensperger et al. (2005) reported a significant prolongation of DA responses to odor at $1 \mathrm{~min}$ following training. We examined the effect of pairing at 10 min following conditioning. Two separate sets of experiments were performed using OCT or $\mathrm{MCH}$ as the CS.

In the upper stalk area, the response to OCT remained unchanged after naïve or US-only pairing, but decreased significantly after forward, backward, and CS-only pairing (Figure 8B), suggesting that the 1 min exposure to OCT, whether paired with electric shock or not, has an inhibitory effect on subsequent responses to OCT. A similar effect was observed when $\mathrm{MCH}$ was used for pairing and testing, although the decrease in response to $\mathrm{MCH}$ met significance only in the forward and CS-only groups, but not the backward group (Figure $8 \mathrm{C}$ ). In the $\alpha$ and $\alpha^{\prime}$ tip areas, the effect of pairing was somewhat similar to that in the upper stalk area, in that the response to $\mathrm{MCH}$ decreased, to various degrees, after the forward, backward or CS-only pairing, but showed no decrease after naïve or US-only pairing (Figure 8C). In the lower stalk/junction area, however, there was no significant change in the response to OCT or $\mathrm{MCH}$ after any of the five conditioning protocols. Nor was there any consistent trend that follows a particular stimulus or paradigm (Figures 8B,C). These data demonstrate that there are significant changes in the response of DA neurons to the trained odor in some areas of the mushroom body lobes at $10 \mathrm{~min}$ post-conditioning. The stimulus responses were inhibited in the $\alpha$ tip, $\alpha^{\prime}$ tip, and upper stalk areas, possibly due to prior odor exposure, but remained 
unchanged in the lower stalk/junction area. Therefore, we did not observe any significant change that correlates specifically to forward pairing. Furthermore, we did not see an obvious change in the time course of odor responses after pairing (not shown).

\section{DISCUSSION}

\section{THE DA NEUROMODULATORY NETWORK IN THE ADULT FLY BRAIN}

Nässel and Elekes (1992) examined the distribution and morphology of neurons exhibiting DA and tyrosine hydroxylase immunoreactivity in blowflies. They characterized 15 clusters of putative DA neurons with respect to cell body location, number of cells per cluster, and areas of projection (Table 1). Seven of the clusters were found in the protocerebrum. They also examined tyrosine hydroxylase immunoreactive neurons in Drosophila and found that the clusters are similar to those in blowflies. Despite these and other previous studies, the complexity of the DA system has prohibited elucidation of the projection patterns of neurons within individual clusters. We used the TH-GAL4 driver and TH expression patterns combined with clonal analysis using the flip-out method to identify eight putative DA neuron clusters in the protocerebrum and map their projection patterns in the adult brain. A knowledge of the projection patterns of individual DA neurons in the adult brain is essential for precise functional studies of this neuromodulatory network.

Although the putative DA neuron clusters differ in size, shape, location and projection patterns, there exist some common features that are notable. One feature is that many of the neurons extend projections over very long distances relative to the size of the adult brain. For instance, the PAL neurons located near the optic tubercle extend their processes to innervate the contralateral optic tubercle and optic lobe (Figures 2A,B in Supplementary Material). The T1 neurons have their cell bodies in the anterior brain yet extend processes to the posterior brain to innervate the protocerebral bridge (Figures 2O,P in Supplementary Material). A second feature of the DA system is that many clusters consist of subtypes of neurons that have distinct projection patterns. Examples include the PPL1, PPL2ab, PPM2, and PPM3 clusters. A third feature is that a given neuropil may be innervated by multiple clusters. The central complex, for instance, is innervated by PPM3, PPL1, and T1.

\section{DA INNERVATION OF THE MUSHROOM BODY NEUROPIL}

One of the more surprising discoveries is the complexity of DA innervation of the mushroom body neuropil. Past models had envisioned DA inputs as providing uniform innervation of the mushroom bodies to convey US information (Han et al., 1996; Kim et al., 2007). Our results revealed three components of putative DA innervation of the mushroom bodies, the PAM DA neuron-horizontal lobe system, the PPL1 DA neuron-vertical lobe-junction-heel system, and the PPL2ab DA neuron-calyx system. The complexity layers further upon separating the PPL1 system into the subcomponents that innervate different zones of the vertical lobes. The PPL1 neurons that project towards the mushroom body lobes displayed five distinct paths, each involving a specific sub-area of the vertical lobes, the lower stalk/junction, the heel and distal peduncle. In a screen for GAL4 enhancer trap strains that label specific subsets of mushroom body intrinsic and extrinsic neurons (MBINs and MBENs, respectively), Tanaka et al.
(2008) found that lobe-associated MBENs arborize in only specific zones of the lobes and form two to five zones in each lobe. They proposed that the lobes are divided into smaller units for carrying out different functional activities. Our findings on the projection patterns of PPL1 neurons, as well as the functional imaging discussed below, support the idea of lobe subcompartmentation. TH-GAL4 expression in the horizontal lobes was noticeably less widespread and intense than $\mathrm{TH}$ expression in the same area. Coincidentally, significantly fewer PAM neurons expressed $\mathrm{TH}$ GAL4 compared to TH. Although some PAM neurons express TH-GAL4 and innervate the distal tips of the horizontal lobes, the coverage is incomplete. Therefore, past studies of DA function using TH-GAL4 may have underestimated the role of the PAM neuron-horizontal lobe component.

Another unexpected finding we made is that the calyx is innervated by putative DA terminals. We found two types of neurons that are members of the PPL2ab cluster that innervate the calyx and are putatively DA. Previous models suggest that DA neurons influence mushroom body output neurons through synapses in the lobes. Our results suggest that another set of interactions occurs at synapses in the calyx.

Overall, our neuroanatomical results indicate that the circuitry of DA influence on the mushroom bodies is enormously complex. Many of the neurites from the three cell body clusters (PAM, PPL1, PPL2ab) extending into the mushroom body neuropil are varicose and contain boutons (Figures 3F-H, 4A3,B3,C3,D-H, 5A-C,F-J and 6A,C) that may reflect a presynaptic function. This predicts that the neurotransmitter DA is released onto discrete zones of the mushroom body neuropil from the putative presynaptic terminals from different types of $T H$-GAL4 neurons. If so, then where are the postsynaptic regions of the putative DA neurons? We consider three broad possibilities for input regions. First, some TH-GAL4 neuron cell bodies exhibit short and fine processes (Figure $\mathbf{5 K}$ ). It is possible that these processes provide some synaptic or non-synaptic input to the neurons. Second, the primary neurite from the PAM, PPL1, and PPL2ab neurons exhibit branching into fine processes en route to the mushroom body neuropil (Figures 4E,G,H, 5A-C,F-J and 6A-D). These fine processes could provide a dendritic function to the neurons. If so, then large, multiple regions of the adult brain (Table 1, "Main Neuropil Projection"), including the mushroom body satellite area (Figures $\mathbf{3 A - C}$, the area between the vertical and the horizontal lobes; Figures $\mathbf{5 F}-\mathbf{H}$ ), may provide the inputs for DA release in the mushroom body neuropil. Third, it is possible that the TH-GAL4 neurons are both pre- and postsynaptic to the mushroom body neurons, such that mushroom body activity would stimulate the release of DA from the nearby DA terminals and this release would stimulate DA receptors expressed by the mushroom body neurons.

\section{FUNCTIONAL RESPONSES OF DA TERMINALS IN THE MUSHROOM BODY LOBES TO ODOR, SHOCK AND CONDITIONING}

We examined the response of putative DA fibers to electric shock and odor in four different subcompartments of the mushroom body lobes, the tips of $\alpha$ and $\alpha^{\prime}$ lobes, the upper stalk area, and the lower stalk/junction area, each shown to be innervated by a distinct subtype of PPL1 neuron. We discovered that the DA fibers 
exhibit different responses to these stimuli. For instance, the $\alpha$ tip responded much more strongly to electric shock than to $\mathrm{MCH}$, however, the $\alpha^{\prime}$ tip responded much more strongly to $\mathrm{MCH}$ than to electric shock. These observations suggest that the different PPL1 neurons have different functions, with some PPL1 neurons providing strong modulatory input to the mushroom body fibers in the $\alpha$ tip in response to electric shock and other PPL1 neurons providing strong modulatory input to the mushroom body fibers in the $\alpha^{\prime}$ lobe in response to odor stimulation. They also suggest that the different compartments of the mushroom body neuropil have different functions. It is also intriguing that the DA fibers respond to both electric shock and odor stimuli. These findings make clear that the DA fibers cannot be providing information about the US stimulus of electric shock only, as originally envisioned (Han et al., 1996), but must provide a much richer input to the mushroom bodies presumably in support of their function in olfactory conditioning. We do not know which neurons convey information about electric shock and odors to the PPL1 neurons. It is possible that the different response properties of these neurons arise from different synaptic inputs onto their cell bodies or onto the neurite en route to the mushroom body neuropil.

We also examined the effect of pairing an odor with electric shock on subsequent response to the odor. The responses observed were quite variable; the most consistent change was that the odor responses generally decreased after forward conditioning, backward conditioning, and the conditioned stimulus-only in the $\alpha$ tip, $\alpha^{\prime}$ tip, and the upper stalk areas. Since these treatments shared the element of a 1 min odor exposure, it is possible that this exposure caused the subsequent decrease. There is no current understanding of the mechanism for this change or it relevance, if any. There was no such decrease in response in the lower stalk/junction, indicating that DA processes that innervate different sub-areas of the mushroom bodies behave differently following exposure to certain combinations of odor and electric shock.

\section{REFERENCES}

Andretic, R., van Swinderen, B., and Greenspan, R. J.(2005). Dopaminergic modulation of arousal in Drosophila. Curr. Biol. 15, 1165-1175.

Björklund, A., and Dunnett, S. B. (2007). Dopamine neuron systems in the brain: an update. Trends Neurosci. 30, 194-202.

Budnik, V., and White, K. (1988). Catecholamine-containing neurons in Drosophila melanogaster: distribution and development. J. Comp. Neurol. 268, 400-413.

Davis, R.L. (2005). Olfactory memory formation in Drosophila: from molecular to systems neuroscience. Annu. Rev. Neurosci. 28, 275-302.

Foltenyi, K., Andretic, R., Newport, J. W., and Greenspan, R. J. (2007). Neurohormonal and neuromodulatory control of sleep in Drosophila. Cold Spring Harb. Symp. Quant. Biol. 72, 565-571.
Friggi-Grelin, F., Coulom, H., Meller, M., Gomez, D., Hirsh, J., and Birman, S. (2003). Targeted gene expression in Drosophila dopaminergic cells using regulatory sequences from tyrosine hydroxylase. J. Neurobiol. 54, 618-627.

Han, K. A., Millar, N. S., Grotewiel, M. S., and Davis, R. L. (1996). DAMB, a novel dopamine receptor expressed specifically in Drosophila mushroom bodies. Neuron 16, 1127-1135.

Kim, Y. C., Lee, H. G., and Han, K. A. (2007). D1 dopamine receptor dDA1 is required in the mushroom body neurons for aversive and appetitive learning in Drosophila. J. Neurosci. 27, 7640-7647.

Kim, Y. C., Lee, H. G., Seong, C. S., and Han, K. A. (2003). Expression of a D1 dopamine receptor $\mathrm{dDA} 1 / \mathrm{DmDOP} 1$ in the central nervous system of Drosophila melanogaster. Gene Expr. Patterns 3, 237-245.

Riemensperger et al. (2005) studied the activity using a FRETbased calcium reporter of DA terminals in a region that covers parts of the $\alpha$ lobe, $\beta$ lobe and $\gamma$ lobe. They reported that this area responded weakly to odor but strongly to electric shock. They also reported that pairing one of two odors with electric shock prolonged the subsequent calcium response to the paired odor $1 \mathrm{~min}$ after training. They proposed that the prolonged calcium response represents the gain of relevance by a previously neutral stimulus, and that DA neurons thereby predict the reinforcement. We failed to observe any amplitude or time course change in response of the DA neurons 10 min after training that might be indicative of acquired predictive value or salience. However, the two studies are not directly comparable since the response properties were examined at different times after training and different calcium reporters were used.

Our functional imaging was performed in the vertical lobes and junction area, since TH-GAL4 expression in the horizontal lobes does not adequately reproduce the intensity and extent of tyrosine hydroxylase expression in these areas. In addition, the DA innervation in calyx is not dense or intense enough to be visible with our current drivers and reporter.

\section{ACKNOWLEDGEMENTS}

The studies were supported by NS052351 grant and the R. P. Doherty-Welch Chair in Science at the Baylor College of Medicine to R. L. D. The content is solely the responsibility of the authors and does not necessarily represent the official views of the National Institute of General Medical Sciences or the National Institutes of Health.

\section{SUPPLEMENTARY MATERIAL}

The Supplemental Material for this article can be found online at http://www.frontiersin.org/neuralcircuits/paper/10.3389/neuro. 04/005.2009.

Kume, K., Kume, S., Park, S. K., Hirsh, J., and Jackson, F. R. (2005). Dopamine is a regulator of arousal in the fruit fly. J. Neurosci. 25, 7377-7384.

Liu, G., Seiler, H., Wen, A., Zars, T., Ito, K., Wolf, R., Heisenberg, M., and Liu, L. (2006). Distinct memory traces for two visual features in the Drosophila brain. Nature 439 551-556.

Liu, T., Dartevelle, L., Yuan, C., Wei, H., Wang, Y., Ferveur, J. F., and Guo, A. (2008). Increased dopamine level enhances male-male courtship in Drosophila. J. Neurosci. 28 5539-5546.

Nässel, D. R., and Elekes, K. (1992). Aminergic neurons in the brain of blowflies and Drosophila: dopamine- and tyrosine hydroxylase-immunoreactive neurons and their relationship with putative histaminergic neurons. Cell Tissue Res. 267, 147-167.
Neckameyer, W. (1998). Dopamine and mushroom bodies in Drosophila: experience-dependent and -independent aspects of sexual behavior. Learn. Mem. 5, 157-165.

Pendleton, R. G., Rasheed, A., Sardina, T., Tully, T., and Hillman, R. (2002). Effects of tyrosine hydroxylase mutants on locomotor activity in Drosophila: a study in functional genomics. Behav. Genet. 32, 89-94.

Riemensperger, T., Völler, T., Stock, P., Buchner, E., and Fiala, A. (2005). Punishment prediction by dopaminergic neurons in Drosophila. Curr. Biol. 15, 1953-1960.

Schultz, W. (2007). Behavioral dopamine signals. Trends Neurosci. 30 , 203-210.

Schwaerzel, M., Monastirioti, M., Scholz, H., Friggi-Grelin, F., Birman, S., and Heisenberg, M. (2003). Dopamine and octopamine 
differentiate between aversive and appetitive olfactory memories in Drosophila. J. Neurosci. 23, 10495-10502.

Strauss, R. (2002). The central complex and the genetic dissection of locomotor behaviour. Curr. Opin. Neurobiol. 12, 633-638.

Tanaka, N. K., Tanimoto, H., and Ito, K. (2008). Neuronal assemblies of the Drosophila mushroom body. J. Comp. Neurol. 508, 711-755.

Tempel, B. L., Livingstone, M. S., and Quinn, W.G. (1984). Mutations in the dopa decarboxylase gene affect learning in Drosophila. Proc. Natl. Acad. Sci. U.S.A. 81, 3577-3581.

Tully, T., and Quinn, W.G. (1985).Classical conditioning and retention in normal and mutant Drosophila melanogaster. J. Comp. Physiol. 157, 263-277.

Vallone, D., Picetti, R., and Borrelli, E. (2000). Structure and function of dopamine receptors. Neurosci. Biobehav. Rev. 24, 125-132.

Wong, A. M., Wang, J. W., and Axel, R. (2002). Spatial representation of the glomerular map in the Drosophila protocerebrum. Cell 109, 229-241.

Yu, D., Akalal, D. B., and Davis, R. L. (2006). Drosophila alpha/beta mushroom body neurons form a branchspecific, long-term cellular memory trace after spaced olfactory conditioning. Neuron 52, 845-855.

Yu, D., Keene, A. C., Srivatsan, A. Waddell, S., and Davis, R. L. (2005). Drosophila DPM neurons form a delayed and branch-specific memory trace after olfactory classical conditioning. Cell 123, 1-13.

Yu, D., Ponomarev, A., and Davis, R. L. (2004). Altered representation of the spatial code for odors after olfactory classical conditioning. Neuron 42 , 437-449.

Zhang, K., Guo, J. Z., Peng, Y., Xi, W., and Guo, A. (2007). Dopamine-mushroom body circuit regulates saliencybased decision-making in Drosophila. Science 316, 1901-1904.

Conflict of Interest Statement: The authors declare that the research was conducted in the absence of any commercial or financial relationships that could be construed as a potential conflict of interest.
Received: 06 April 2009; paper pending published: 25 April 2009; accepted: 03 June 2009; published online: 01 July 2009.

Citation: Mao $Z$ and Davis RL (2009) Eight different types of dopaminergic neurons innervate the Drosophila mushroom body neuropil: anatomical and physiological heterogeneity. Front. Neural Circuits (2009) 3:5. doi:10.3389/neuro.04.005.2009

Copyright (C) 2009 Mao and Davis. This is an open-access article subject to an exclusive license agreement between the authors and the Frontiers Research Foundation, which permits unrestricted use, distribution, and reproduction in any medium, provided the original authors and source are credited. 